\title{
Adaptive Isotopic Approximation of Nonsingular Curves: the Parameterizability and Nonlocal Isotopy Approach
}

\author{
Long Lin • Chee Yap
}

Received: 2 July 2009 / Revised: 16 March 2010 / Accepted: 23 April 2010 /

Published online: 25 March 2011

(C) Springer Science+Business Media, LLC 2011

\begin{abstract}
We consider domain subdivision algorithms for computing isotopic approximations of a nonsingular algebraic curve. The curve is given by a polynomial equation $f(X, Y)=0$. Two algorithms in this area are from Snyder (1992) SIGGRAPH Comput. Graphics, 26(2), 121 and Plantinga and Vegter (2004) In Proc. Eurographics Symposium on Geometry Processing, pp. 245-254. We introduce a new algorithm that combines the advantages of these two algorithms: like Snyder, we use the parameterizability criterion for subdivision, and like Plantinga and Vegter, we exploit nonlocal isotopy. We further extend our algorithm in two important and practical directions: first, we allow subdivision cells to be rectangles with arbitrary but bounded aspect ratios. Second, we extend the input domains to be regions $R_{0}$ with arbitrary geometry and which might not be simply connected. Our algorithm halts as long as the curve has no singularities in the region, and intersects the boundary of $R_{0}$ transversally. Our algorithm is practical and easy to implement exactly. We report some very encouraging experimental results, showing that our algorithms can be much more efficient than the algorithms of Plantinga-Vegter and Snyder.
\end{abstract}

Keywords Meshing · Curve approximation · Isotopy · Parameterizability · Subdivision algorithms $\cdot$ Topological correctness $\cdot$ Exact algorithms

This work is supported by NSF Grants CCF-0728977 and CCF-0917093.

L. Lin $(\bowtie) \cdot$ C. Yap

Courant Institute of Mathematical Sciences, New York University, 251 Mercer Street, New York, NY 10012, USA

e-mail: 1lin@cs.nyu.edu

C. Yap

e-mail: yap@cs.nyu.edu 


\section{Introduction}

Approximation of curves and surfaces is a basic problem in many areas such as simulation, computer graphics, and geometric modeling. The approximate surface is often a triangulated surface, also known as a mesh. See the recent book [5] for an algorithmic perspective on meshing problems. We focus on curves, and in this case the "mesh" is just a (planar) straightline graph $G$ (or PSLG, see [21]). Our problem is this: given a region $R_{0} \subseteq \mathbb{R}^{2}$ of interest, an error bound $\varepsilon>0$, and a curve $S$ implicitly represented by an equation $f(X, Y)=0$, to find a piecewise linear $\varepsilon$-approximation $G$ of $S \cap R_{0}$.

The correctness criteria for $G$ has two parts: topological correctness and geometric accuracy. Geometric accuracy is typically taken to mean that the Hausdorff distance between $G$ and $S \cap R_{0}$ is at most $\varepsilon$. In recent years, the topological correctness is understood to mean that the approximate curve $G$ should be isotopic to $S \cap R_{0}$; see [2] for further discussion of isotopy. Correspondingly, the meshing problem can be solved in two stages: first we produce an output $\widetilde{G}$ that is isotopic to $S \cap R_{0}$. Subsequently, we refine $\widetilde{G}$ into a graph $G$ with the requisite geometric accuracy. We may call these the isolation and refinement stages, following a terminology used for the $1 \mathrm{D}$ analogue of root approximation. The isolation stage is more challenging and usually draws most of the attention in algorithms literature. Most of our emphasis is also on the isolation stage.

There are three general approaches to meshing problems: algebraic, geometric, or numeric. Algebraic approaches are based on polynomial operations and algebraic number manipulation. Most algebraic algorithms can be reduced to the powerful tool of cylindrical algebraic decomposition (CAD) [1], but such methods are too inefficient, even in the plane. This has led to much interest in numerical algebraic methods (e.g., [14]). But for special cases such as quadric surfaces [24] or cubic curves [12], efficient algebraic algorithms have been devised. Geometric approaches exploit geometric properties such as Morse theory [3, 28] or Delaunay triangulations [10]. These geometric properties are encoded into the primitives used by the algorithm. Typical primitives include the orientation predicates or ray shooting operations. Numeric approaches focus on approximation and numerical primitives such as function evaluation $[15,20]$. Such primitives are usually embedded in simple global iterative schemes such as bisection. There is considerable work along this line in the interval arithmetic community (e.g., Martin et al. [16]). These algorithms are often called "curve tracing algorithms." See Ratschek and Rokne [23] for references to curve tracing papers. Until recently, numeric approaches were shunned by computational geometers as lacking exactness or complexity analysis. This is unfortunate as practitioners overwhelmingly favor numeric approaches for two simple reasons: they are efficient and easy to implement. Our overall goal is to address the above shortcomings of numerical approaches while retaining their advantages. Clearly, some algorithms are best viewed as hybrids of these approaches. All three approaches are exemplified in the survey [2].

As suggested above, geometric algorithms are usually described in an abstract computational model that postulates certain geometric primitives (i.e., operations or predicates). These primitives may be implemented either by numerical or algebraic techniques; the algorithm itself is somewhat indifferent to this choice. For the 


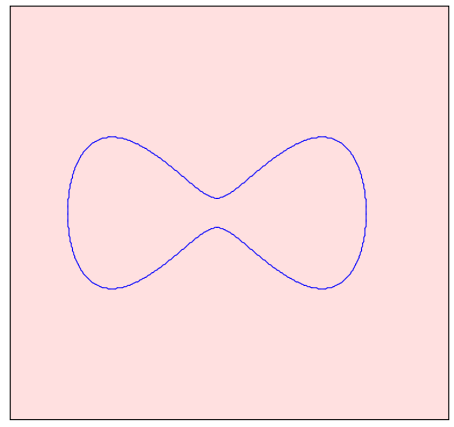

(a) Original Curve

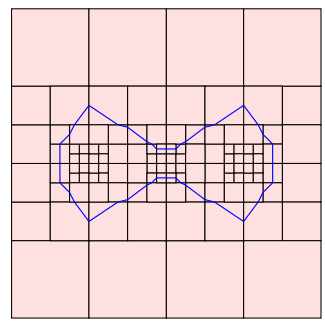

(b) Snyder

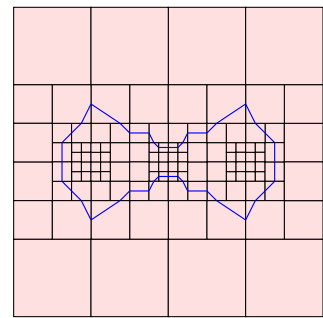

(d) Balanced Cxy

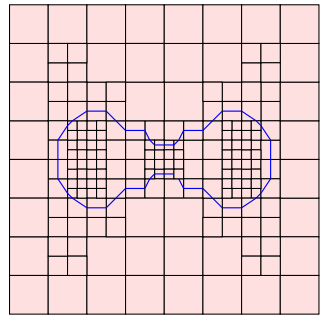

(c) PV

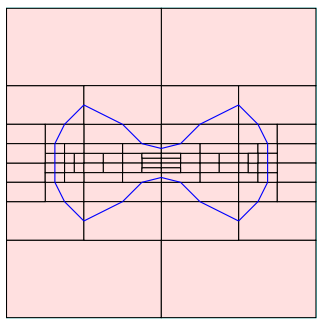

(e) Rectangular Cxy

Fig. 1 Domain Subdivision Approaches to approximating the curve $f(X, Y)=X^{2}(1-X)(1+X)-Y^{2}+$ $0.01=0$ : comparison of four algorithms

meshing problem, a popular approach is based on sampling points on input surface $[2,4,10]$. The geometric primitive here is ray-shooting; it returns the first point (if it exists) that the ray intersects on the input surface. For algebraic surfaces, this primitive reduces to a special case of real root isolation (namely, finding the smallest positive real root). The sampled points have algebraic number coordinates. In addition, the algorithms typically maintain a Delaunay triangulation of the sampled points and thus would need orientation predicates on algebraic points. But exact implementation of these primitives requires expensive and nontrivial algebraic number manipulations. This does not seem justified in meshing applications. On the other hand, if we use approximations for sample points, these may no longer lie on the surface. This gives rise to the well-known "implementation gap" concerns of computational geometry [29]: nonrobustness, degeneracies, approximation, etc. In contrast, the subdivision methods studied in this paper suffers no such implementation gaps. As subdivision methods are important to large communities of practitioners in numerical scientific computation, it behooves us to develop such methods into exact and quantifiable tools for geometric algorithms.

\subsection{Recent Progress in Subdivision Algorithms}

In this paper, we focus on algorithms based on domain ${ }^{1}$ subdivisions methods. Figure 1 illustrates the output of four such algorithms on the input curve $f(X, Y)=$ $X^{2}(1-X)(1+X)-Y^{2}+0.01=0$. These output are from our implementation of

\footnotetext{
${ }^{1}$ We use the term "domain subdivision" to refer to the subdivision of the underlying space $\mathbb{R}^{2}$ or $\mathbb{R}^{3}$ in which the curve or surface lives. Subdivision can also take place in parameter space, as in Bezier surfaces.
} 
the algorithms of Snyder and of Plantinga and Vegter (PV), and two new algorithms of this paper (Balanced Cxy, Rectangular Cxy).

We view subdivision algorithms as falling under the numeric approaches (see below for the numerical computational model). The simplest form of domain subdivision use only axes-parallel boxes (e.g., in bisection searches and Marching Cube [15]). According to a taxonomy of meshing algorithms in [2], this form is called "cube-based scaffolding." The scaffolding provides a global data structure, but the implementation of the primitives must still be reduced to algebraic or numerical operations. For example, Seidel and Wolpert [25] used algebraic primitives within this scaffolding. Our algorithms will focus on numerical primitives. Note that numerical primitives are not necessarily immune to implementation gaps. For instance, the Morse theory approach to surface meshing in [28] reveals such gaps.

The direct precursors for our work are the subdivision algorithms of Plantinga and Vegter [20] and Snyder [27]. Both algorithms are based on interval arithmetic [18] and the ability to evaluate the exact sign of function $f(X, Y)$ at bigfloat values. For a large class of functions $f(X, Y)$, not necessarily algebraic, these primitives can be easily implemented exactly using a bigfloat number package. Snyder's algorithm is applicable in all dimensions (but it has termination problems as noted below); currently, the Plantinga-Vegter method is only known in two and three dimensions. Ben Galehouse [13] has a subdivision algorithm for meshing surfaces in any dimension, but like Snyder, he requires recursive meshing of the boundary. All these algorithms are also related to the SCCI-hybrid algorithm for curve tracing by Ratschek and Rokne [23].

The problem of approximating curves defined by a bivariate polynomial is the two-dimensional version of the general problem of approximating the hypersurface defined by a $d$-variate polynomial. The case $d=3$ is clearly very important in practice. When $d=1$, this is the classic root approximation problem. Computing up to isotopy in this case is known as the root isolation problem. There is considerable recent progress in subdivision methods for root isolation: for a nice survey of work on the Descartes-Bernstein methods, including the so-called bit-stream algorithms, see [11]; for results related to the continued fraction method, see [26].

Both Plantinga-Vegter and Snyder assume that the input curves and surfaces are nonsingular. Recently, numerical subdivision algorithms that can work with nonsingularities and degeneracies have appeared: in [30], we gave a Bezier curve intersection algorithm that is correct even in the presence of tangential intersection. Subdivision techniques for approximating curves with isolated singularities were given in [6]. The paper also extended the algorithm of Plantinga and Vegter to domains with irregular geometry. In [8], we introduced the 1D versions of the Plantinga-Vegter algorithm, and extending it to treat singularities (i.e., multiple zeros). A key attraction of subdivision algorithms is their adaptive complexity. In [7], we introduced continuous and algebraic amortization techniques, resulting in one of the first adaptive analysis of subdivision algorithms.

\subsection{Contributions of this Paper}

The present paper represents a more practical contribution to the preceding series of development: we break no new ground in terms of doing something we could not do 
before. On the other hand, we introduce implementable ideas that make subdivision algorithms more useful than ever, while remaining theoretically sound.

Our main contribution is a new approach, and a corresponding new meshing algorithm, that combines the relative advantages of Snyder and Plantinga-Vegter: we retain the weaker $C_{x y}$-predicate of Snyder, but like Plantinga and Vegter, we do not require local isotopy. Our processing of each box is just as simple as in PV. However, achieving geometric accuracy is somewhat harder with the $C_{x y}$-predicate. We will address this issue separately.

In this paper, we give the first complete proof of the global isotopy of the Plantinga-Vegter method. Such a proof, being global, is more subtle than the correctness of Snyder's parameterizability approach (which is entirely local).

When meshing a curve that is almost horizontal in some neighborhood, it is very useful to allow boxes in that neighborhood to be elongated along the horizontal direction. Note that the PV algorithm limits the aspect ratios of boxes to be less than 2. So another contribution is to allow subdivision boxes with variable but bounded aspect ratio. The aspect ratio of a box is the length of the longest side of a box over that of its shortest side. This further improves the adaptivity of our method. Other practical improvements include allowing domains of arbitrary geometry, as in [6]. Thus the input domains need not be connected or simply connected.

We have implemented our algorithms, and to perform comparisons, we have also implemented the Plantinga-Vegter and Snyder algorithms. We will provide experimental evidence showing that our new approach can greatly speed up the previous algorithms. See Fig. 1 for some impressions of our approach: our Balanced Cxy Algorithm produces fewer boxes than Plantinga and Vegter, but unlike Snyder, we achieved this without having to isolating roots. The Rectangular Cxy Algorithm produces even fewer boxes than Snyder's algorithm. An important assumption is made in our comparisons: we assume that the goal is to compute the correct isotopy-type, with no concern for geometric accuracy (i.e., we set the parameter $\varepsilon \infty$ ). The output from Plantinga-Vegter or Snyder would be expected to have better geometric accuracy. Our assumption is consistent with the corresponding assumption in the one-dimensional problem, where the focus is on root isolation, not on root refinement. Indeed, once we have the roots isolated or the isotopy-type computed, there might well be better techniques for geometric refinement.

\section{Overview of Subdivision Algorithms}

To provide intuitions for our new results, we will recall the works of Snyder and Plantinga-Vegter. In most of our discussion, we fix a real curve

$$
S:=f^{-1}(0)=\left\{p \in \mathbb{R}^{2}: f(p)=0\right\},
$$

which is specified by a $C^{1}$ function, $f(X, Y): \mathbb{R}^{2} \rightarrow \mathbb{R}$. We assume interval arithmetic and interval versions of functions such as $f$ and its partial derivatives $f_{x}, f_{y}$.

A box is given by $B=I \times J \subseteq \mathbb{R}^{2}$ where $I, J$ are real intervals. Let $m(I)$ and $w(I)$ denote the midpoint and width of $I$. For a box $B=I \times J$, let $w_{x}(B):=w(I)$, 
$m_{x}(B)=m(I)$; similarly for $w_{y}(B), w_{y}(B)$. Then the midpoint, width, and diameter of $B$ are (resp.) $m(B):=\left(m_{x}(B), m_{y}(B)\right), w(B):=\min \left\{w_{x}(B), w_{y}(B)\right\}$, and $d(B):=\max \left\{w_{x}(B), w_{y}(B)\right\}$. We name the four sides and corners of a box $B$ by their compass directions (north, south, east, west and NE, NW, SW, SE). We say that $B$ has uniform sign if the input function $f$ has the same sign at each of its four corners. If $p, q \in \mathbb{R}$ are the SW and NE corners of $B$, we may denote $B=[p, q]$. A full-split of $B$ is to subdivide $B$ into four equal subboxes; a half-split subdivides $B$ into two equal subboxes. There are two kinds of half-splits: horizontal and vertical. These subboxes are called the children of $B$. If the children of the full-split of $B$ are denoted $B_{1}, \ldots, B_{4}$ (with $B_{i}$ in the $i$ th quadrant relative to $m(B)$ ), then the children in a horizontal (resp., vertical) half-split are $B_{12}, B_{34}$ (resp., $B_{14}, B_{23}$ ), where $B_{i j}=B_{i} \cup B_{j}$. We use the side/corner terminology for boxes but reserve the edge/vertex terminology for the approximation straightline graphs $G$ (or PSLG [21]).

\subsection{Our Computational Model}

To see why our algorithms are free of implementation gaps, we take a closer look at the computational model we need. Bigfloats or dyadic numbers is the set $\mathbb{F}=\mathbb{Z}[1 / 2]=\left\{m 2^{n}: m, n \in \mathbb{Z}\right\}$. All numerical computations in our algorithms will be reduced to exact ring operations $( \pm, \times)$ and comparisons on bigfloat numbers. Bigfloat number packages are efficient and widely available (e.g., GMP, LEDA, or Core Library). More generally, $\mathbb{F}$ can be replaced by any "computational ring" [31] satisfying some basic axioms to support exact real approximation.

We also use interval arithmetic [18] — the main tool being inclusion functions [22]. An inclusion function for $f(X, Y)$ is a function $\square f(I, J)=\square f(B)$ that takes input intervals and returns an interval that satisfies the inclusion property: $f(B)=\{f(x, y)$ : $(x, y) \in B\} \subseteq \square f(B)$. We call $\square f$ a box function for $f$ if, in addition, it is point convergent, i.e., for any strictly decreasing sequence $B_{0} \supset B_{1} \supset \cdots$ of boxes that converges to a point $p$, we have $\square f\left(B_{i}\right) \rightarrow p$ as $i \rightarrow \infty$. For our computational model, it is assumed that the input arguments to $\square f$ are dyadic boxes, and it returns a dyadic box. We also need box versions of the derivatives $f_{x}, f_{y}$.

As in [6], we call $f$ a PV function if $f: \mathbb{R}^{2} \rightarrow \mathbb{R}$ is $C^{1}$, there exist computable box functions $\square f, \square f_{x}, \square f_{y}$, and the sign of $f$ at dyadic points $p \in \mathbb{F}^{2}$ is computable. It will be clear that the algorithms of this paper can be easy to implement with no numerical errors when the input $f$ is a PV function, and all numerical inputs are dyadic. Therefore, nonrobustness issues are moot. See [6, 22] for additional information.

In contrast to our computational model, the standard model of numerical analysis only supports inexact arithmetic (up to unit round-off error). This leads to the implementation gap issues mentioned in the introduction. Such a model is assumed by Ratschek and Rokne, and even though they have the same basic approach as this paper, they had to discuss rounding errors [23, §2.5]. Moreover, in their model, computing the sign of $f(X, Y)$ at a point $p=\left(x_{0}, y_{0}\right)$ is problematic.

\subsection{Generic Subdivision Algorithm}

The subdivision algorithms in this paper have a simple global structure. Each algorithm has a small number of steps called phases. Each phase takes an input queue $Q$ 
and returns some output data structure, $Q^{\prime}$. Note that $Q^{\prime}$ need not be a queue, but $Q$ is always a queue of boxes. Each phase is a while-loop that extracts a box $B$ from $Q$, processes $B$, possibly reinserting children of $B$ back into $Q$. The phase ends when $Q$ is empty. If $Q^{\prime}$ is a queue of boxes, it could be used as input for the next phase. We next describe a generic algorithm with three phases: Subdivision, Refinement, and Construction.

For the Subdivision Phase, the input $Q_{\text {in }}$ and output $Q_{\text {out }}$ are both queues holding boxes. The subdivision depends on two box predicates, $C_{i n}(B)$ and $C_{\text {out }}(B)$. For each box $B$ extracted from $Q_{\text {in }}$, we first check if $C_{\text {out }}(B)$ holds. If so, $B$ is discarded. Otherwise, if $C_{\text {in }}(B)$ holds, then insert $B$ into $Q_{\text {out }}$. Otherwise, we full-split $B$ and insert the children into $Q_{\text {in }}$. Next, the Refinement Phase takes the output queue from the Subdivision Phase and further subdivides the boxes to satisfy additional criteriathese refined boxes are put in an output queue $Q_{\text {ref }}$. Strictly speaking, it should be possible to combine refinement with the subdivision phase. Finally, the Construction Phase takes $Q_{r e f}$ as its input and produces an output structure $G=(V, E)$ representing a planar straight line graph. As we process each box $B$ in the input queue, we insert vertices and edges into $V$ and $E$, respectively.

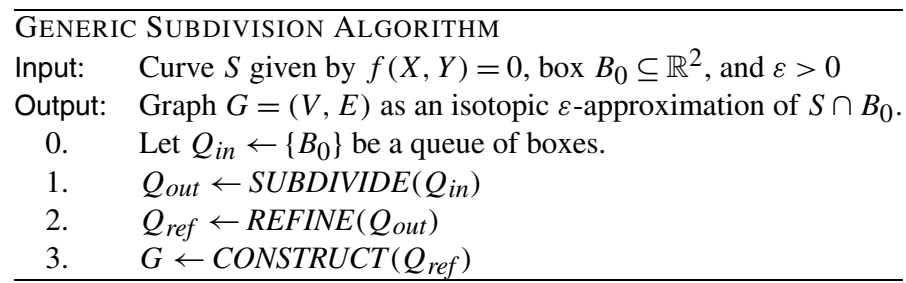

\subsection{Example: Crude Marching Cube}

Let us instantiate the generic algorithm just described, to produce a crude but still useful algorithm for "curve tracing" (cf. [16]). For the Subdivision Phase, we must specify two box predicates: let the $C_{\text {out }}$ predicate be instantiated as

$$
C_{0}(B): 0 \notin \square f(B) \text {. }
$$

If $C_{0}(B)$ holds, clearly the curve $S$ does not pass through $B$, and $B$ may be discarded. Let $C_{\text {in }}$ predicate be instantiated by $C_{\varepsilon}(B)$ which states that the sides of $B$ have lengths less than some $\varepsilon>0$. Thus, all the boxes in output $Q_{\text {out }}$ have width $\leq \varepsilon$. The current Refinement Phase does nothing (so $Q_{\text {ref }}=Q_{\text {out }}$ ). For the Construction Phase, we must specify how to process each box $B \in Q_{\text {ref }}$. The goal is to create vertices to be inserted into $V$ and create edges (which are straightline segments joining pairs of vertices) to be inserted into $E$. The output is a straightline graph $G=(V, E)$.

We construct $G$ as follows: for each $B \in Q_{\text {ref }}$, we evaluate the sign of $f$ at each of the four corners of $B$. If the endpoints of a side of $B$ have different signs, we introduce a vertex $v \in V$ at the midpoint of the side. (Of course, if $v$ has already been created while processing a neighboring box of $B$, we do not duplicate $v$.) Clearly, $B$ has 0 , 2 , or 4 vertices on its sides. If $B$ has two vertices, we introduce an edge to connected them (see Fig. 2(a), (b)). These edges represent two types of connected components 


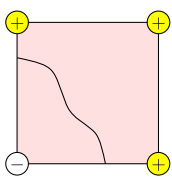

(i)

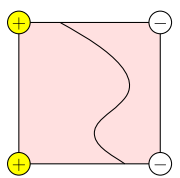

(ii)

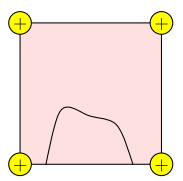

(iii)

\section{KEY: \\ Corner: $\oplus \quad \ominus$ Vertex:}

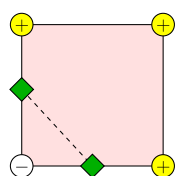

(a)

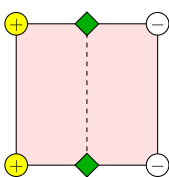

(b)

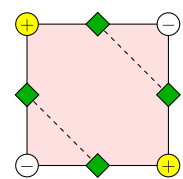

(c)

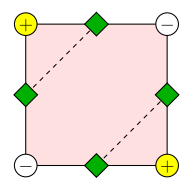

(d)

Fig. 2 Components Types: (i) corner, (ii) cut, (iii) incursion. Simple Connection Rules: (a), (b) corner and cut edge; (c), (d) double corner edges

of $S \cap B$ : corner and cut components (respectively) as illustrated in Fig. 2(i), (ii). A third type of connected component is an incursion (or $B$-incursion) (Fig. 2(iii)) is not represented, but omission can be justified by isotopy (the reduction step in Fig. 4(i), (ii)). If $B$ has 4 vertices, we introduce two pairs of nonintersecting edges to connect them (see Fig. 2(c), (d)); there are two ways to do this, but we choose either one arbitrarily. In general, the corners of $B$ may have a zero sign. But henceforth, we give them an arbitrary sign (say, positive). This can be justified by isotopy, as in [20].

This completes our description of a crude Marching Cube algorithm. Other subdivision algorithms to be discussed will be seen as refinements of this crude algorithm. The output graph $G=(V, E)$ is an approximation to $S \cap B_{0}$, up to " $\varepsilon$ resolution." If $\varepsilon$ is screen resolution, this is adequate for the purposes of graphical display. Martin et al. [16] gave a comparative study of various numerical implementations of the box predicates $C_{\text {out }}, C_{\text {in }}$.

\subsection{Snyder's Parameterizability Approach}

Our crude Marching Cube makes no claims on topological correctness. Until recently, no numerical subdivision algorithms can promise much better. In particular, the ability to handle singularities is regarded as an open problem for numerical methods [2, p. 182]. But many papers assume manifolds in order to avoid singularity. In the present paper, we only assume that the curve $S$ has no singularities in the region $R_{0}$ of interest. More precisely, $f^{2}+f_{x}^{2}+f_{y}^{2}$ does not vanish at any point in $R_{0}$. Our main issue is to ensure isotopy in such a situation. In domain subdivision, two related approaches have been introduced by Snyder [27] and Plantinga and Vegter [20]. In Snyder's approach, the predicate $C_{i n}$ is chosen to be

$$
C_{x y}(B): C_{x}(B) \vee C_{y}(B),
$$

where $C_{x}(B)$ is the predicate $0 \notin \square f_{x}(B)$, and similarly for $C_{y}(B)$ with respect to $f_{y}$. A curve $S$ is said to be parameterizable in the $x$-direction (or, $x$-parameterizable) 
Fig. 3 The box components of a $C_{y}$-box

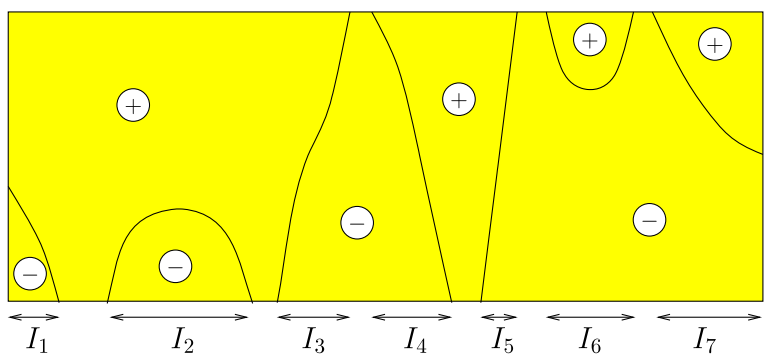

in a box $B$ if each vertical line intersects $S \cap B$ at most once. Clearly, $C_{y}(B)$ implies that $S$ is $x$-parameterizable in $B$; this is illustrated in Fig. 3. During the Construction Phase, we isolate the intersections of $S$ with the boundary $\partial B$ of each box $B \in Q_{\text {ref }}$ (this amounts to root isolation). With sufficient root refinement, we would be able to correctly construct the isotopy type of $S \cap B$. Note that this isotopy type can be arbitrarily complex, as seen in Fig. 3.

\subsection{Plantinga and Vegter's Small Normal Variation Approach}

Unfortunately, Snyder's algorithm (assuming that the method is recursively applied to the boundary of $B$ ) may not terminate ${ }^{2}$ if the curve intersects $\partial B$ tangentially [2, p. 195] (e.g., $f=x^{2}+y^{2}-1$, and $B_{0}:=[(-2,-2),(2,2)]$; Snyder's algorithm would keep subdividing in boxes containing the point $(1,0))$. In view of this, the credit for the first complete subdivision algorithm to achieve isotopic approximation of nonsingular curves and surfaces belongs to Plantinga and Vegter [20]. In place of $C_{x y}(B)$, the Plantinga-Vegter (or PV) algorithm uses a stronger predicate that we denote by $C_{1}(B)$ :

$$
C_{1}(B): 0 \notin\left(\square f_{x}(B)\right)^{2}+\left(\square f_{y}(B)\right)^{2}
$$

It important that the operation $[a, b]^{2}$ of squaring an interval $[a, b]=\square f_{z}(B)(z \in$ $\{x, y\})$ in (4) is defined as [min $\left.a^{2}, a b, b^{2}, \max a^{2}, a b, b^{2}\right]$ and not as [0, $\left.\max a^{2}, b^{2}\right]$. This predicate is called the "small normal variation" condition in [2]. To see that $C_{1}(B)$ implies $C_{x y}(B)$, we can follow [20] by rewriting (4) as

$$
0 \notin\langle\square \nabla f(B), \square \nabla f(B)\rangle,
$$

where $\nabla f(p):=\left(f_{x}(p), f_{y}(p)\right)$ denotes the gradient at a point $p, \square f(B):=$ $\left(\square f_{x}(B), \square f_{y}(B)\right)$, and $\langle\cdot, \cdot\rangle$ is just the scalar product of two vectors. This shows that if $p, q \in B$, then $\langle\nabla f(p), \nabla f(q)\rangle>0$. Suppose that some $p \in B$ has a vertical gradient (there are two choices, up or down). Then no $q \in B$ can have a horizontal gradient (there are two choices, left or right). We conclude that $f^{-1}(0) \cap B$ is parameterizable in the $x$-direction. There is a symmetric argument in which the roles of

\footnotetext{
${ }^{2}$ In meshing curves, one can handle this problem by some root isolation method that handle multiple roots, but the problem is more serious in meshing surfaces.
} 
horizontal and vertical directions are interchanged. The PV algorithm has a remarkable nonlocal isotopy property:

\section{It does not guarantee isotopy of the approximation $G$ with the curve $S$} within each box $B \in Q_{\text {ref }}$.

We view this property favorably because local isotopy in each $B$ is seen as an artifact of the subdivision scheme and could greatly increase the number of subdivisions. The nontermination of Snyder's algorithm is precisely because it insists on local isotopy. The processing of $C_{1}$-boxes is extremely simple as compared to Snyder's approach. In fact, it is a slight extension of the connection rules in our crude Marching Cube above (see Fig. 7). This advantage shows up even more in 3D, where Snyder's algorithm must recursively solve the $2 \mathrm{D}$ isotopy problem on the boundary of each subdivision box. On the negative side, $C_{1}(B)$ is a stronger predicate than $C_{x y}(B)$ and may cause more subdivisions than $C_{x y}(B)$. In view of these tradeoffs, it is not immediately clear which approach is more efficient.

\subsection{Quadtrees}

Instead of queues, we prefer to work with a slightly more elaborate structure: a quadtree is a rooted tree $T$ whose nodes $u$ are associated with boxes $B(u)$ and if $u$ is an internal node, then it either has four or two children whose associated boxes are obtained by full- or half-splitting $B(u)$. Two nodes $u, v$ are said to be adjacent (or neighbors) if the interiors of $B(u)$ and $B(v)$ are disjoint, but their boundary overlap. Overlapping means that $B(u) \cap B(v)$ is a line segment, not just a point or empty. In order for $T$ to represent regions of fairly complex geometry, we assume that each leaf of $T$ is tagged with a Boolean flag, "in" or "out." So we may speak of the inleaves or out-leaves of $T$. The associated boxes are called in-boxes or out-boxes. The quadtree $T$ represents a region denoted $R(T) \subseteq \mathbb{R}^{2}$ which is just the union of all the in-boxes. Following [6], we call $R(T)$ a nice region. The notion of side/corner is relative to a box $B$.

A refinement step is an operation on a quadtree $T$ in which we split any in-leaf $u \in T$, tagging the children as in or out. Note that we do not split out-leaves. Although the original tagging is arbitrary, subsequent tagging of new nodes created by refinement must follow a fixed rule, depending on some fixed pair of box predicates $\pi=\left(\pi_{\text {in }}, \pi_{\text {out }}\right)$. We tag a node as "out" if its associated box $B$ satisfies $\pi_{\text {out }}(B)$, else it is "in." If $\pi_{i n}(B)$ holds but not $\pi_{\text {out }}(B)$, we say that $B$ is terminal. In this paper, $\pi_{\text {out }}$ is always the predicate $C_{0}$ above; it ensures that out-boxes can safely be omitted in our approximation of the curve $S$. So we only focus on $\pi_{i n}$.

To recap, our algorithm begins with a nice region $R(T)$ in which the tagging of nodes of $T$ are arbitrarily assigned. Subsequently, we refine $T$ using the above rules for tagging new nodes. Thus, there are two types of "out" leaves: original or $C_{0}$, and two types of "in" leaves: terminal or nonterminal.

A refinement of $T$ is obtained by a sequence of refinement steps. Note that if $T^{\prime}$ is a refinement of $T$, then $R\left(T^{\prime}\right) \subseteq R(T)$. We are interested in three properties of quadtrees $T$, each obtained by successive refinements: 
- $S U B D I V I D E_{\pi_{i n}}(T)$ returns a quadtree that satisfies the pair $\pi=\left(\pi_{i n}, C_{0}\right)$ of box predicates, i.e., each out-box $B$ is either originally tagged as "out" or else $C_{0}(B)$ holds, and each in-box must satisfy $\pi_{i n}$, but not $C_{0}$.

- $R E G U L A R I Z E(T)$ returns a regular quadtree, i.e., any two adjacent in-boxes have the same depth. Thus,

$$
R E G U L A R I Z E(T) \equiv S U B D I V I D E_{\pi_{r e g}}(T),
$$

where $\pi_{r e g}(B) \equiv$ all in-boxes adjacent to $B$ have width $\geq w(B)$. Note that we must not replace the condition "width $\geq w(B)$ " by "width $=w(B)$ " because this can cause the smallest square to split and possibly lead to nontermination. In contrast to Plantinga and Vegter's notion of regularity which requires all the leaves to have the same depth, ours allow the leaves of different connected components of $R(T)$ to have different depths.

- $B A L A N C E(T)$ returns a balanced quadtree, i.e., one where the depths of any two adjacent in-boxes differ by at most one. Thus,

$$
B A L A N C E(T) \equiv S U B D I V I D E_{\pi_{b a l}}(T),
$$

where $\pi_{b a l}(B) \equiv$ all in-boxes adjacent to $B$ have width $\geq \frac{1}{2} w(B)$.

A useful terminology is the notion of "segments" of a quadtree $T$. Roughly speaking, segments are the units into which a side of a box is subdivided. There are two types of segments: a boundary segment $e$ is a side of an in-box of $T$ such that $e \in \partial R(T)$; an internal segment $e$ has the form $e=B \cap B^{\prime}$, where $B, B^{\prime}$ are adjacent in-boxes of $T$. Thus each side of a box in $T$ is divided into one or more segments. If $T$ is a regular quadtree, then each side of an in-box of $T$ is also a segment; if $T$ is a balanced quadtree, then each side of an in-box of $T$ is composed of either one or two segments. A boundary box is an in-box that has a boundary segment as one of its sides.

For now, assume that the above three subroutines use only full-splits; the general case, where we also allow half-splits, is treated in Section 7. Given a quadtree $T$, we assume a simple subroutine $Q \leftarrow \operatorname{InBox}(T)$ that returns a queue $Q$ containing all the in-boxes in $T$. Thus, the above three subroutines can be viewed as "phases" (see Sect. 2.2) whose input queues are $\operatorname{InBox}(T)$. These subroutines are easily implemented by a simple while-loop as described earlier.

\subsection{Perturbation}

The correctness statements of geometric algorithms can be quite involved in the presence of degeneracy. To avoid such complications, and in the spirit of exploiting nonlocal isotopy, we exploit perturbations of $f$. We call $\tilde{f}: \mathbb{R}^{2} \rightarrow \mathbb{R}$ a nice perturbation of $f: \mathbb{R}^{2} \rightarrow \mathbb{R}$ relative to $T$ if

(i) $\tilde{f}^{-1}(0) \cap \operatorname{Interior}(R(T)) \approx \tilde{f}^{-1}(0) \cap R(T)$.

(ii) $\forall \epsilon>0, \exists f_{\epsilon}: \mathbb{R}^{2} \rightarrow \mathbb{R}$ such that (a) $\left|f(q)-f_{\epsilon}(q)\right|<\epsilon$ for $\forall q \in \mathbb{R}^{2}$, and (b) $\tilde{f}(p) f_{\epsilon}(p)>0$ for any corner $p$ of $T$. 
An intuitive way to get a nice perturbation of $f$ is to slightly shift the $S=f^{-1}(0)$ so that the resulting curve does not pass any of the corners of the boxes in $T$.

Lemma 1 For any given $f$ and $T$, there exists an nice perturbation $\tilde{f}$ of $f$ relative to $T$.

We need not explicitly $\tilde{f}$ (which depends on $T$ which is being expanded during the algorithm). Instead, each time we evaluate $f$ at a corner $p$ of a subdivision box, if $f(p)=0$, then we simply declare the sign to be positive. We justify this by saying that we are really using a nice perturbation $\tilde{f}$ instead of $f$. Of course, we could give $f$ any nonzero sign at each $p$, as long as the sign is treated consistently for each $p$. This use of $\tilde{f}$ incurs no additional cost or complexity for our algorithm. For notational simplicity, we simply refer to the some nice perturbation $\tilde{f}$ as $f$.

\section{Regular Cxy Algorithm}

In this paper, we will describe three increasingly sophisticated subdivision algorithms for curves, all based on the $C_{x y}$ predicate. These will be known as the Regular Cxy, Balanced Cxy, and Rectangular Cxy Algorithms. For the first two algorithms, we only perform full-splits of boxes. We now present the first of these three algorithms.

Our initial goal is to replace the $C_{1}$-predicate in the PV Algorithm by the parameterizability condition of Snyder. As in Plantinga and Vegter [20], we first consider a simplified version in which we regularize the quadtree, i.e., reduce all adjacent inboxes to the same depth. This is our Regular Cxy Algorithm, which has this form:

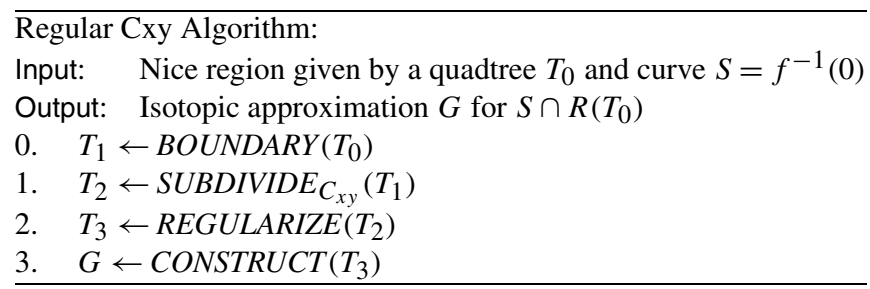

Note that there are four phases (Phases 0 to 3 ) and only Phase 0 remains to be clarified. Suppose that we ignore Phase 0 (treating the operation $\operatorname{BOUNDARY}\left(T_{0}\right)$ as a no-op). Then the algorithm is just an elaboration of the Crude Marching Cube, in which we replace its (empty) Refinement Phase by a Regularization Phase, and replace the predicate $C_{\varepsilon}$ by $C_{x y}$. The Construction Phase here is simpler than in the Crude Marching Cube because we never have four vertices on the sides of an in-box in view of condition $C_{x y}(B)$. Thus, the only connection rules we need are Fig. 2(a), (b) (i.e., Fig. 2(c), (d) are excluded).

The naive correctness statement is this: " $S \cap R\left(T_{0}\right)$ is isotopic to $G$." But this naive statement may fail because of "incursions" or "excursions" at boundary boxes. More precisely, suppose that $B$ is a boundary box and let $e \subseteq \partial R\left(T_{0}\right)$ be a segment of $B$. We say $S$ makes an incursion (resp., excursion) at $e$ if $S \cap B$ (resp., $S \cap\left(\mathbb{R}^{2} \backslash R\left(T_{0}\right)\right)$ ) has a connected component $C$ with both end points in $e$. Thus, $C$ enters and exits $B$ 
(resp., exits and reenters $B$ ) at $e$. Such incursions/excursions are not captured by our output graph $G$. So a nontrivial Phase 0 is necessary to fix this problem.

\subsection{Boundary Processing}

The role of Phase 0 is to "secure" the original boundary of $R\left(T_{0}\right)$. This basically amounts to isolating all the intersections of $S$ with $\partial R\left(T_{0}\right)$. In principle, we could invoke any exact root isolation algorithm for this purpose. However, we prefer to apply the same subdivision method, albeit applied recursively to one dimension lower. In general, for a $d$-dimensional subdivision algorithm, we want to recursively use the $(d-1)$-dimensional analogue for processing its boundary. For 1D, this algorithm is essentially the EVAL algorithm for real root isolation [7, 8, 17].

The basic idea is to keep splitting any boundary box that has a potential incursion or excursion. Initially place all the boundary boxes of $T_{0}$ into a queue $Q_{0}$, and while $Q_{0}$ is nonempty, we remove a boundary box $B$ and "tests" each of its boundary segment $e$ (there may be one to four such segments). It $e$ fails the test, it is split, and its boundary children is put back into $Q_{0}$. If each boundary segment of $B$ passes the "test," we discard $B$ (i.e., it does not have to be split). But this amounts to doing nothing.

Let us now clarify the "test" on a boundary segment $e$. The 1D analogue of $C_{0}$ and $C_{x y}$ predicates are (respectively)

$$
C_{0}^{\prime}(e): 0 \notin \square f(e), \quad C_{x y}^{\prime}(e): 0 \notin \square f_{z}(e),
$$

where $z=x$ if $e$ is horizontal, and $z=y$ if $e$ is vertical. If $C_{0}^{\prime}(e)$ holds, the curve does not intersect $e$. If $C_{x y}^{\prime}(e)$ holds, then there can be no incursion/excursion curve at $e$. We say that $e$ fails the test if either $C_{0}^{\prime}(e)$ or $C_{x y}^{\prime}(e)$ does not hold. When $Q_{0}$ is empty, we terminate Phase 0 . The output from this Phase is a quadtree $T_{1}$ that refines the boundary boxes of $T_{0}$ so that the curve $S$ intersects each boundary segment of $R\left(T_{1}\right)$ at most once.

There is only one problem: if the curve intersects the boundary of $R\left(T_{0}\right)$ tangentially, this method does not terminate. This problem was addressed by [6], using a weakened correctness statement and a more elaborate algorithm. For this paper, we shall be contented with the above simple solution, but we need to make the strong requirement that any intersection of the input curve with the boundary of $R\left(T_{0}\right)$ must be transversal. By definition, transversal intersection does not allow the curve to just touch a corner of $R\left(T_{0}\right)$ without entering the interior of $R\left(T_{0}\right)$.

\subsection{Correctness}

It is perhaps surprising that this simple algorithm, only a small extension of Crude Marching Cube, already produces the correct isotopy. Because it is easy to implement, it may have credible practicality.

Theorem 2 (Correctness of Regular Cxy Algorithm) The algorithm terminates, provided that $S$ intersects $\partial R\left(T_{0}\right)$ only transversally and $f$ is nonsingular inside $R\left(T_{0}\right)$. Moreover, the output graph $G$ is isotopic to $S \cap R\left(T_{0}\right)$. 
The proof will be spread over several steps. We first prove termination. Only the boundary and subdivision phases have the potential for non-termination. The following lemma provides the condition to guarantee their termination.

\section{Lemma 3}

(i) If $S=f^{-1}(0)$ intersects the boundary of $R\left(T_{0}\right)$ only transversally, then the Boundary Phase will terminate.

(ii) If $f$ has no singularities in $R\left(T_{0}\right)$, then the Subdivision Phase will terminate.

Proof (i) If the Boundary Phase does not terminate, then there is an infinite decreasing sequence of sides, $e_{0} \supset e_{1} \supset \cdots$, such that each $C_{0}^{\prime}\left(e_{i}\right)$ and $C_{x y}^{\prime}\left(e_{i}\right)$ fail. Without loss of generality, let $e_{0}$ be horizontal and $e_{i} \rightarrow p$ as $i \rightarrow \infty$. Then $C_{x y}^{\prime}\left(e_{i}\right)$ failing means that $0 \in \square f_{x}\left(e_{i}\right)$. Since $\square f_{x}\left(e_{i}\right) \rightarrow f_{x}(p)$, we conclude that $f_{x}(p)=0$. Similarly, $C_{0}^{\prime}\left(e_{i}\right)$ failing implies $f(p)=0$. This shows that $f^{-1}(0)$ intersects $e_{0}$ tangentially.

(ii) If the Subdivision Phase does not terminate, then there is an infinite decreasing sequence of boxes $B_{0} \supset B_{1} \supset \cdots$ such that each $C_{0}\left(B_{i}\right)$ and $C_{x y}\left(B_{i}\right)$ fails. Thus,

$$
0 \in\left(\square f\left(B_{i}\right) \cap \square f_{x}\left(B_{i}\right) \cap \square f_{y}\left(B_{i}\right)\right) .
$$

The boxes $B_{i}$ must converge ${ }^{3}$ to some point $p \in R\left(T_{0}\right)$ as $i \rightarrow \infty$. Since $\square f$ is a box function for $f$, we conclude that $\square\left(B_{i}\right) \rightarrow f(p)$. Then (6) implies $0=f(p)=$ $f_{x}(p)=f_{y}(p)$. Thus, $f$ is singular in $R\left(T_{0}\right)$.

\section{Partial Correctness of Regular Cxy Algorithm}

The basic partial correctness technique in Plantinga and Vegter [20] is to apply isotopies which remove any excursion of the curve $f^{-1}(0)$ from a box $B$ to its neighboring box $B^{\prime}$. Such isotopies are not "local" to any single box, but it is nevertheless still fairly local, being restricted to a union $B \cup B^{\prime}$ of two adjacent boxes. But in our algorithm, an excursion from $B$ can pass through a sequence of boxes, so we need a more global view of how to apply such isotopies.

We next prove partial correctness: if the algorithm terminates, the output $G$ is isotopic to $S \cap R\left(T_{0}\right)$. The key idea in the proof is to use isotopy to transform the curve $S \cap R\left(T_{0}\right)=S \cap R\left(T_{3}\right)$ repeatedly, until we finally obtain a curve $S^{*}$ that we can show is isotopic to $G$. Each transformation step removes a pair of intersections between $S$ and the boundary of boxes, as illustrated in Fig. 4(i), (ii): the pair $\left(a^{\prime}, b^{\prime}\right)$ is eliminated via the isotopic transformation from (i) to (ii). We say that the pair $\left(a^{\prime}, b^{\prime}\right)$ is reducible. We will make this precise.

\footnotetext{
${ }^{3}$ The existence of $p$ depends only on the existence of a bound $r$ on the maximum aspect ratio-so this proof applies in the more general setting of Rectangular Cxy Algorithm later.
} 


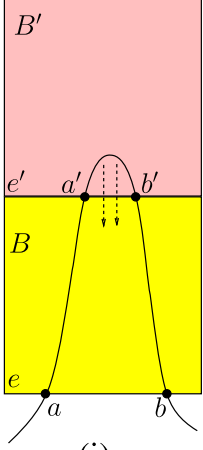

(i)

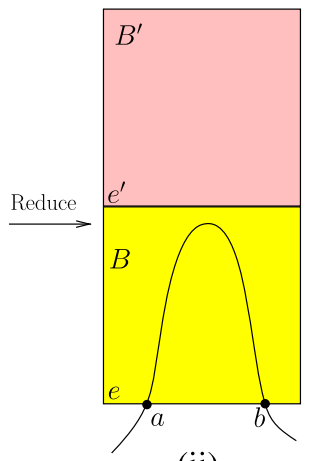

(ii)

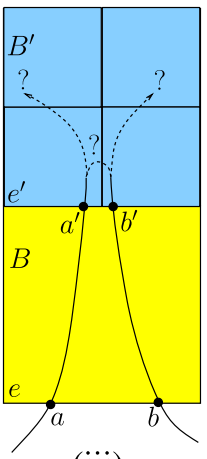

(iii)

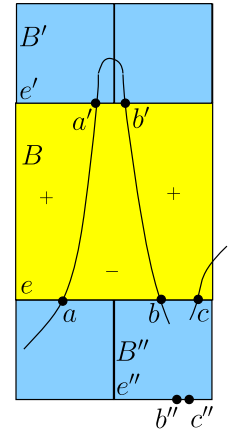

(iv)

Fig. 4 Reduction step with $\left(a^{\prime}, b^{\prime}\right) \prec(a, b)$

\subsection{Partial Ordering of Convergent Pairs}

To give a structure for our induction, we need a partial ordering on pairs of intersection points, such as $(a, b)$ or $\left(a^{\prime}, b^{\prime}\right)$ in Fig. 4(i), (ii). If $a=\left(a_{x}, a_{y}\right), b=\left(b_{x}, b_{y}\right)$ are points, it is convenient to write " $a<_{x} b$ " to mean that $a_{x}<b_{x}$. Similarly, $a<_{y} b$ means that $a_{y}<b_{y}$. Also, $a \leq_{x} b$ means that $a_{x} \leq b_{x}$.

Let $e$ be a segment, so $e=B \cap B^{\prime}$ for some in-boxes $B$ and $B^{\prime}$ (see Fig. 4(i)). Assume that $C_{x y}$ holds at $B$ and $B^{\prime}$. By symmetry, assume that $e$ is a horizontal segment (the following definitions can be modified if $e$ is vertical).

Consider the set $S \cap e$. By our assumption that $S$ has no vertical or horizontal components, $S \cap e$ is a finite set. In general, $S$ can intersect $e$ at points with multiplicity greater than 1; then, as in [9], we can view $S \cap e$ as a multiset where each point $p \in S \cap e$ has multiplicity 1 or 2 , according as $S$ intersects $e$ with odd or even multiplicity. However, we can avoid this complication by simple perturbation arguments (this will be noted in the proof below). Therefore, we assume that $S$ intersects $e$ transversally. Let $S \cap e=\left\{p_{1}, \ldots, p_{m}\right\}$ where the points are sorted so that $p_{1}<_{x} p_{2}<_{x} \cdots<_{x} p_{m}$. A pair of the form $\left(p_{i}, p_{i+1}\right)$ is called a consecutive pair of $e$. Clearly, $e$ contains a consecutive pair iff $m \geq 2$. Moreover, if $m \geq 2$ and $C_{x y}(B)$ holds, then $S$ must be $x$-parameterizable in $B$.

A consecutive pair $(a, b)$ of a horizontal segment $e$ is said to be upward convergent if the two portions of the curve $S$, near $a$ and near $b$ (respectively), are moving closer to each other as the respective curve portions move upward across $e$. This is equivalent to saying that the slope of the curve $S$ is positive at $a$ and negative at $b$. This is illustrated in Figs. 4(i) and (ii).

We have three related definitions: if $(a, b)$ is a consecutive pair of segment $e$, we say that $(a, b)$ is downward convergent if $e$ is a horizontal segment and the slope of $f$ is negative at $a$ and positive at $b$. If $e$ is a vertical segment, we similarly define left or right convergent. A key property is the following:

Lemma 4 Let $e=B \cap B^{\prime}$ be a segment. If $B$ and $B^{\prime}$ satisfies $C_{x y}$, then every consecutive pair of e is convergent (upward or downward or left or right). 
Proof Without loss of generality, let $e$ be horizontal, and $(a, b)$ be a consecutive pair of $e$. We must show that $e$ is either upward or downward convergent. Since $C_{x y}(B)$ holds, the fact that $f^{-1}(0)$ intersects $e$ in two distinct points $a, b$ means that, in fact, $C_{y}(B)$ holds. Without loss of generality, assume that $f_{y}(B)>0$. There are two possibilities: $f((a+b) / 2)>0$ or $f((a+b) / 2)<0$. In the former case, we have $f_{x}(a)>0$ and $f_{x}(b)<0$, and so the slope of $f^{-1}(0)$ is negative at $a$ and positive at $b$. This means that $(a, b)$ is downward convergent. The latter case will imply that $(a, b)$ is upward convergent.

By symmetry, we mainly focus on upward convergent pair $(a, b)$ of a horizontal segment $e=B \cap B^{\prime}$. Because of the presence of $(a, b)$, the curve $S$ is $x$ parameterizable in $B$ and $B^{\prime}$; so $C_{y}$ must hold at $B$ and at $B^{\prime}$. Without loss of generality, we henceforth assume that $f_{y}(B)>0$ and $f_{y}\left(B^{\prime}\right)>0$.

Let $P=P(f)$ be the set of all upward convergent pairs of segments in the quadtree $T_{3}$. Note that none of these pairs lies on a boundary segment because of the Boundary Processing Sect. 3.1. Let $X_{a}$ be the connected component of $B \cap S$ that contains $a$; similarly for $X_{b}$. Let $a^{\prime}$ be the other endpoint of $X_{a}$; similarly for $b^{\prime}$. In case $X_{a}=X_{b}$, we have that $a^{\prime}=b$ and $b^{\prime}=a$ and that $X_{a}$ is a $B$-incursion. Hence we call $(a, b)$ an incursion pair (see Fig. 4(ii)). But suppose that $X_{a} \neq X_{b}$; then $X_{a}$ and $X_{b}$ are cut components (see Fig. 4(i)) satisfying

$$
a<_{x} a^{\prime}<_{x} b^{\prime}<_{x} b
$$

because $C_{y}$ holds in $B$. This is illustrated in Fig. 4(i).

Also, it is easy to see that $f_{x}\left(a^{\prime}\right)<0$ and $f_{x}\left(b^{\prime}\right)>0$. Clearly $S$ intersects the relative interior of the line segment $\left[a^{\prime}, b^{\prime}\right]$ an even number of times. If there are $2 k \geq 0$ such intersections, then we can find $k+1$ convergent pairs on $\left[a^{\prime}, b^{\prime}\right]$. Suppose that these pairs are

$$
\left(a_{0}, b_{0}\right),\left(a_{1}, b_{1}\right), \ldots,\left(a_{k}, b_{k}\right)
$$

where $a_{0}=a^{\prime}$ and $b_{k}=b^{\prime}$. Then we define

$$
\left(a_{i}, b_{i}\right) \prec(a, b)
$$

for each $i=0, \ldots, k$. Let $\preceq$ denote the reflexive, transitive closure of the set of binary relations defined as in (7). It is easy to see that $\preceq$ is a partial order on $P$. For regularized quadtrees, the minimal elements of this partial order are those $(a, b)$ for which $X_{a}=X_{b}$ are incursion components or boundary pairs; for balanced quadtrees (next section), this is no longer true.

\subsection{Compatibility}

So far, our box predicates $C_{0}, C_{1}, C_{x y}$ and Phases such as $\operatorname{CONSTRUCT(T)}$ are implicitly based on some PV function $f$. In order to explicitly indicate their dependence on $f$, we put $f$ in the superscript as in $C_{0}^{f}, C_{1}^{f}, C_{x y}^{f}, \operatorname{CONSTRUCT}^{f}(T)$.

Let $T$ be a quadtree, and $f, g$ be PV functions. We say that $f$ is compatible with $T$ if for each boundary segment $e$ of $T$, the curve $f^{-1}(0)$ intersects $e$ at most once, 
and any intersection is transversal. If $f$ and $g$ are both compatible with $T$, and for all corners $u$ of in-boxes, we have $f(u) g(u)>0$, then we say that $f$ and $g$ are consistent on $T$.

The role of the 0th and 1st Phases of the Regular Cxy Algorithm is to construct a quadtree that is compatible with $f$ and to make the curve $x$ - or $y$-monotone inside each box of $T$. Recall that $\operatorname{CONSTRUCT}^{f}(T)$ produces a straightline graph $G=$ $(V, E)$ where, for each segment $e$ of $T$, we introduce a vertex $v \in V$ iff the $f$ has opposite signs at the endpoints of $e$, and for each in-box with two vertices $u, v$ on its boundary, we introduce an edge $(u, v) \in E$.

Lemma 5 Let $T$ be a quadtree, and $f$ be a $P V$ function. If $T$ is regular and compatible with $f$, then the graph $G:=\operatorname{CONSTRUCT}^{f}(T)$ is isotopic to $f^{-1}(0) \cap R(T)$.

Proof We will inductively define a sequence $f_{0}, f_{1}, f_{2}, \ldots, f_{n}$ of $C^{1}$ functions such that $f_{0}:=f$ and each pair $f_{i-1}, f_{i}$ is consistent over $T(i=1, \ldots, n)$ and $S_{i} \approx S_{i-1}$, where $S_{i}:=f_{i}^{-1}(0)$.

We may ensure that each $S_{i}$ intersects the segments of $T$ only transversally and avoids the corners of in-boxes. Hence, we can define the partial ordering $P_{i}=P\left(f_{i}\right)$ of upward convergent pairs (relative to the segments of quadtree $T$ ). The transformation from $S_{i}$ to $S_{i-1}$ is illustrated by the "reduction step" of Fig. 4(i), (ii) and amounts to the removal of an upward convergent pair which is minimal in the partial order $P_{i}$. No other convergent pairs of $P_{i-1}$ are affected by this transformation. It is then clear that $S_{i} \approx S_{i-1}$. Thus, we have the further property that $P_{i} \subseteq P_{i-1}$ with $\left|P_{i}\right|=\left|P_{i-1}\right|-1=\left|P_{0}\right|-i$. We stop after $n=\left|P_{0}\right|$ transformations, when $\left|P_{n}\right|=0$.

We can similarly remove all the downward, left and right convergent pairs, by repeating the preceding process three more times. We finally arrive at a function $\bar{f}$ such that there are no consecutive pairs on any segment. According to Lemma 4, this means that the curve $\bar{S}:=\bar{f}^{-1}(0)$ intersects each segment at most once. Moreover, the curves $\bar{S}$ and $S=f^{-1}(0)$ are isotopic.

It remains to show that $\bar{S} \cap R(T) \simeq G$, where $G=\operatorname{CONSTRUCT}^{f}(T)$. Let $B$ be any in-box of $T$. Since $C_{x y}^{f}(B)$ holds, our construction of $G$ ensures that $|G \cap \partial B| \in$ $\{0,2\}$. Note that $G$ has a vertex at a segment $e$ iff $|\bar{S} \cap e|=1$. Since we may assume that $\bar{S}$ does not intersect the corners of $B$, it follows that $|G \cap \partial B|=|\bar{S} \cap \partial B|$. In other words, $G \cap \partial B$ is isotopic to $\bar{S} \cap \partial B$. Moreover, this can be extended into an isotopy for the entire in-box: $G \cap B$ is isotopic to $\bar{S} \cap B$.

The transformation of the function $f_{i-1}$ into $f_{i}$ can be made explicit if desired. Suppose that the transformation removes the $\preceq$-minimal upward convergent pair $(a, b)$ on segment $e$. Let $e=B \cap B^{\prime}$, where $B, B^{\prime}$ are in-boxes, and $B$ lies north of $e$. We emphasize that this transformation is local to $B \cup B^{\prime}$. Let $X_{a, b}$ denote the connected component of $S_{i-1} \cap B$ whose endpoints are $a, b$. Let $B_{a, b}$ denote the smallest rectangle that contains $X_{a, b}$. Suppose $B_{a, b}=\left[x_{1}, x_{2}\right] \times\left[y_{1}, y_{2}\right]$. For $\epsilon>0$, let $B_{a, b}^{\epsilon}=\left[x_{1}-\epsilon, x_{2}+\epsilon\right] \times\left[y_{1}-\epsilon, y_{2}+\epsilon\right]$. Choose $\epsilon$ sufficiently small so that $B_{a, b}^{\epsilon} \cap S_{i-1}$ is comprised of a unique component, denoted $X_{a, b}^{\epsilon}$. Now define $f_{i}:\left[x_{1}-\epsilon, x_{2}+\epsilon\right] \times\left[y_{1}-\epsilon, y_{2}+\epsilon\right] \rightarrow \mathbb{R}$ so that $f_{i}$ is the identity on the boundary of $\left[x_{1}-\epsilon, x_{2}+\epsilon\right] \times\left[y_{1}-\epsilon, y_{2}+\epsilon\right]$, but otherwise $f_{i}(x, y)=f_{i-1}(x, g(x, y))$, where 
the function $g(x, y)$ has the property that $g(x, \cdot)$ is a piecewise linear shear. Explicit formulas for $g$ can be given if desired. Moreover, $f_{i}(x, y)=0$ implies $y<y_{1}$. In other words, $f_{i}^{-1}(0) \cap\left[x_{1}-\epsilon, x_{2}+\epsilon\right] \times\left[y_{1}-\epsilon, y_{2}+\epsilon\right]=f_{i}^{-1}(0) \cap\left[x_{1}-\epsilon, x_{2}+\right.$ $\epsilon] \times\left[y_{1}-\epsilon, y_{1}\right]$. Thus the component $X_{a, b}^{\epsilon}$ has moved out of $B$ into $B^{\prime}$. Finally, let extend the function $f_{i}$ to all of the Euclidean plane by defining $f_{i}(x, y)=f_{i-1}(x, y)$ for all $(x, y) \notin\left[x_{1}-\epsilon, x_{2}+\epsilon\right] \times\left[y_{1}-\epsilon, y_{2}+\epsilon\right]$.

Corollary 6 Let $T$ be a regularized quadtree. If $f, g$ are consistent on $T$, then $f^{-1}(0) \cap R(T) \approx g^{-1}(0) \cap R(T)$.

Proof Note that the consistency of $f$ and $g$ implies that $\operatorname{CONSTRUCT}^{f}(T)=$ $\operatorname{CONSTRUCT}^{g}(T)$. By the previous lemma, we also have $f^{-1}(0) \cap R(T) \approx$ $\operatorname{CONSTRUCT}^{f}(T)$ and $g^{-1}(0) \cap R(T) \approx \operatorname{CONSTRUCT} T^{g}(T)$.

\section{Conclusion of the Proof of Theorem 2}

Proof Termination follows from Lemma 3. We note how each phase of the Regular Cxy Algorithm provides the necessary properties for correctness: Phase 0 converts $T_{0}$ to $T_{1}$ which satisfies the Boundary Condition for compatibility between $T_{1}$ and $f$. Phase 1 converts $T_{1}$ to $T_{2}$ which satisfies the Box Condition for compatibility between $T_{2}$ and $f$ (the boundary condition is preserved in this transformation). So $T_{2}$ is compatible with $f$. Phase 2 converts $T_{2}$ into a regular quadtree, again preserving compatibility. Note that $f^{-1}(0) \cap R\left(T_{0}\right)=f^{-1}(0) \cap R\left(T_{3}\right)$, since the out-boxes introduced by each of these phases satisfy $C_{0}$. By Lemma 5 , the output $G$ from Phase 3 is isotopic to $f^{-1}(0) \cap R\left(T_{3}\right)$.

\section{Balanced Cxy Algorithm}

The Regular Cxy Algorithm is nonadaptive because of regularization. The PV Algorithm is similar to the Regular Cxy Algorithm, except that we replace the Regularization Phase by a Balancing Phase and use $C_{1}$ predicate instead of $C_{x y}$. The connection rules in the Construction Phase will become only slightly more elaborate (see below and $[6,20])$.

\subsection{Issue of Ambiguous Boxes}

We now explore the possibility of using the $C_{x y}$ predicate in the PV Algorithm. To indicate the critical issue, consider a horizontally stretched hyperbola $(c Y+X) \times$ $(c Y-X)=1$ for some $c \gg 1$ as in Fig. 5(a). We run the PV algorithm on this input hyperbola and a quadtree $T$ where $R(T)=[(-5,-1),(11,15)]$, which is a $16 \times$ 16 square. It is conceivable the Subdivision Phase ends up discarding all subboxes except for the eight shaded squares inside $[(-5,-1),(5,1)]$, as shown in Fig. 5(b). Moreover, each of the four larger squares $\left(B_{1}, B_{2}, B_{1}^{\prime}, B_{2}^{\prime}\right)$ satisfies $C_{x}$, while the four smaller squares satisfy $C_{y}$. The four smaller squares were split from a larger square $[(-1,-1),(1,1)]$, which does not satisfy $C_{x y}$. The output graph $G$ obtained 
(a)

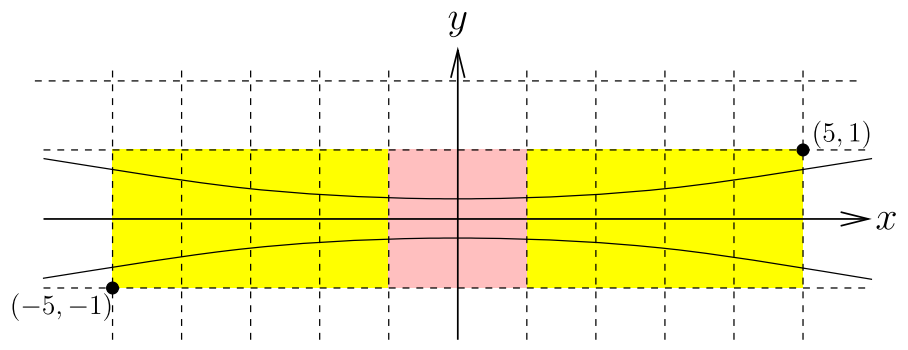

(b)

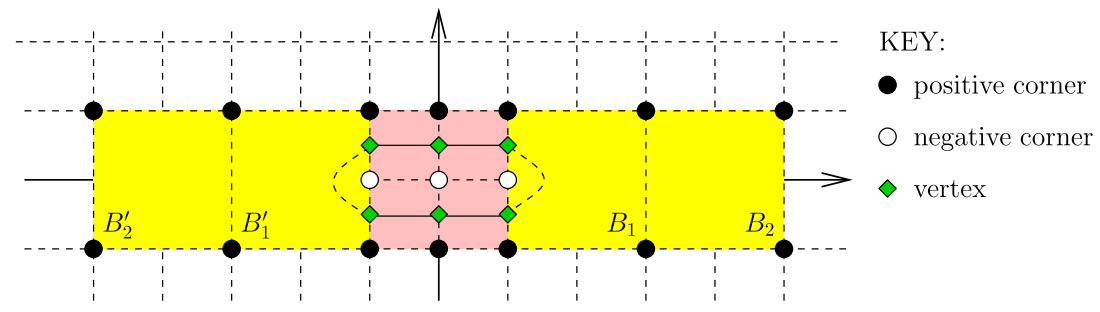

Fig. 5 (a) Input "flat" hyperbola. (b) Output graph with wrong isotopy type

by using the connection rules of Fig. 7 is the 6-vertex graph shown in Fig. 5(b). Since $G$ forms a loop, it is clearly wrong. The error occurred in the boxes $B_{1}$ (and by symmetry, in $B_{1}^{\prime}$ ), where $G \cap B_{1}$ has only one connected component, while $S \cap B_{1}$ has two components. If we had split $B_{1}$, we would have discovered that there are two, not one component, in $S \cap B_{1}$. The box $B_{1}$ (and $B_{1}^{\prime}$ ) is said to be "ambiguous." In general, a leaf box $B$ is ambiguous if it (i) satisfies $C_{x y}$, (ii) has uniform sign at its corners, and (iii) has exactly two vertices. The ambiguity classification marks $B$ for a full-split. A slightly more elaborate definition can be provided to avoid unnecessary splits. ${ }^{4}$

Figure 6(a) shows an ambiguous box $B$ (it satisfies $C_{y}$ but not $C_{x}$ ). Note that our definition of ambiguity does not depend on whether $B$ 's north or south sides have been subdivided. If we full-split box $B$, the situation resolves into one of two possibilities, as in Fig. 6(b) or 6(c). In fact, Fig. 6(c) has two subcases, depending on the sign of the midpoint of the box. In any case, splitting an ambiguous box will "disambiguate" it. In case of Fig. 6(b), this might further cause the eastern neighbor of $B$ to become ambiguous. This propagation of ambiguity can be iterated any number of times. But propagation of splitting can be caused also by the need to rebalance boxes. However, both kinds of propagation will terminate because if a box splits, it is "caused" by a neighboring box of smaller size. In our hyperbola example in Fig. 5(b), the splitting of $B_{1}$ and $B_{1}^{\prime}$ will cause $B_{2}$ and $B_{2}^{\prime}$ to become ambiguous and be split. The final output graph will now be correct.

\footnotetext{
${ }^{4}$ That is, we may require an optional condition: (iv) If $B$ satisfies $C_{y}$ (resp., $C_{x}$ ), then one of its horizontal (resp., vertical) sides has not yet been subdivided.
} 


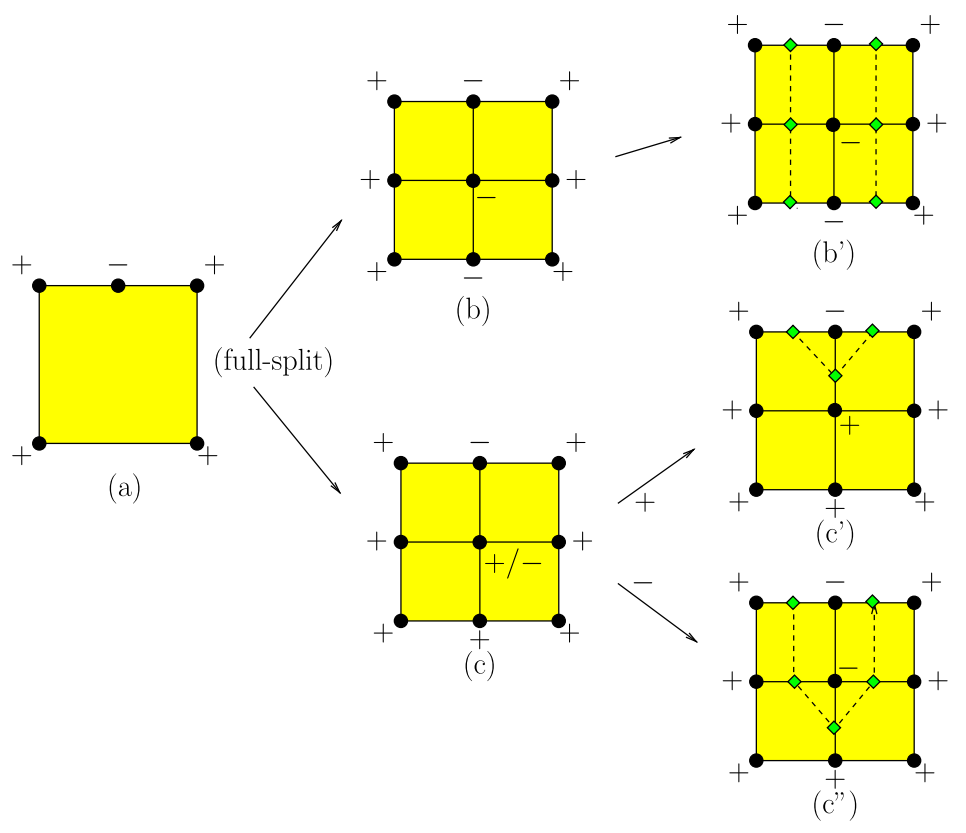

Fig. 6 Ambiguous box (a) and its resolution (b'), (c'), (c')

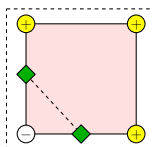

(a)

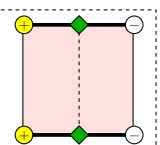

(b)

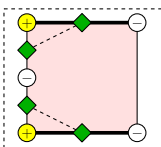

(c)

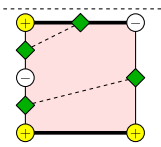

(d)

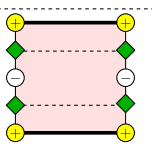

(e)

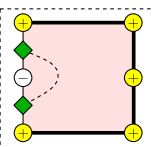

(f)

Fig. 7 Extended Connection Rules: Cases (c)-(f) treats two vertices lying on one side of a box

\subsection{The Algorithm}

We now present the overall algorithm using our (now familiar) 4 Phases. To propagate and resolve ambiguity, we need a slightly more elaborate Construction Phase, which we call CONSTRUCT ${ }^{+}$in the following:

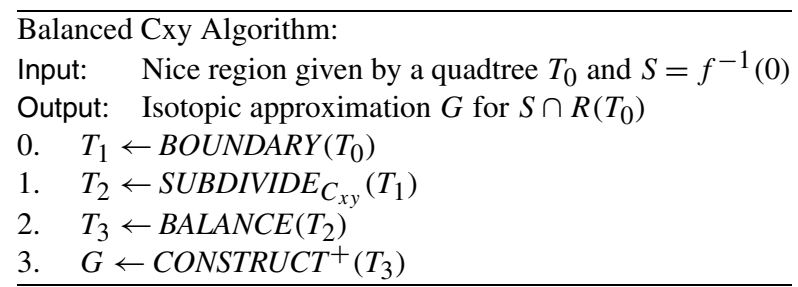

The first three phases are now standard. Our goal in the CONSTRUCT ${ }^{+}\left(T_{3}\right)$ is to do the usual construction of the graph $G=(V, E)$, but also to disambiguate boxes. 
As usual, the input quadtree $T_{3}$ for CONSTRUCT $T^{+}$provides a queue $Q$ of in-boxes to be processed. However, the queue is now a priority queue. The priority of a box $B$ is given by the inverse of its width (i.e., smaller-width boxes have higher priority), and among those boxes with the same width, the ambiguous boxes have higher priority. We may organize this priority queue as a list $Q=\left(L_{1}, L_{2}, \ldots\right)$ of sublists. Each sublist $L_{i}$ contains all the in-boxes of a given width (boxes in $L_{i}$ has width half of those in $L_{i+1}$ ). In each sublist, the ambiguous boxes appear ahead of the nonambiguous boxes. Note that some sublists may be empty. It is easy to manipulate these lists: when a box is removed from $L_{i}$ to be split, its children go into sublist $L_{i+1}$. If a box in $L_{i}$ becomes ambiguous because of insertion of two new vertices on one of its sides, it is moved to the front of its sublist. The top-of-queue is the first element in the first nonempty list $L_{i}$.

We need two subroutines called

\section{$\operatorname{REBALANCE}(B), \quad \operatorname{PROCESS}(B)$.}

To "rebalance" $B$, we split any neighbor of $B$ whose width is more than twice that of $B$ and recursively rebalance the children of its split neighbors. These children are reinserted into the queue for future processing. More precisely:

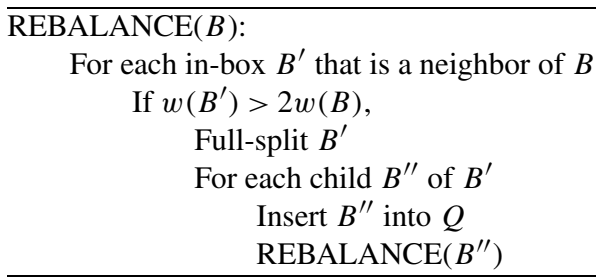

To "process" $B$, we add vertices to the sides of $B$ (if they were not already added) and connect them according to the following rules: as shown in the next section, $B$ has 0,2 , or 4 vertices on its boundary. If $B$ has 2 vertices, we connect them as for the crude Marching Cube Fig. 2(a), (b), but reproduced in Fig. 7(a), (b). If $B$ has 4 vertices, it turns out that two of them will lie on one side of $B$; we connect these two vertices to the other two in such a way that the edges are nonintersecting (this connection rule is unique, unlike Fig. 2(c), (d)). These rules are summarized in Fig. 7(a)-(f).

Four new cases arise Fig. 7(c)-(f). Case (e) does not arise in the original PV algorithm. Case (f) does arise in PV, but it is ambiguous and so will be eliminated by our algorithm through its disambiguating process. Thus, case (f) does not ${ }^{5}$ arise in our current algorithm.

It is easy to see that these cases are exhaustive, and they can occur. There is an additional detail: if we add new vertices, we must also update the priority of any in-box neighbor of $B$ that may become ambiguous as a result. More precisely:

\footnotetext{
${ }^{5}$ Note that case (f) may arise if our definition of ambiguity includes the optional condition (iv).
} 
PROCESS $(B)$ :

For each side of $B$,

If it has not been split, has not yet been processed, and has

a change in sign at its endpoints,

Add a vertex

Update the priority of its neighbor (if an in-box) across

this side.

Connect the (at most four) vertices in the sides of $B$ using the connection rules of Fig. 2(a), (b) and Fig. 7(a)-(d).

The correctness of PROCESS $(B)$ depends on the fact that any smaller box has already been processed. Moreover, $B$ itself is terminal (will not be split in the future).

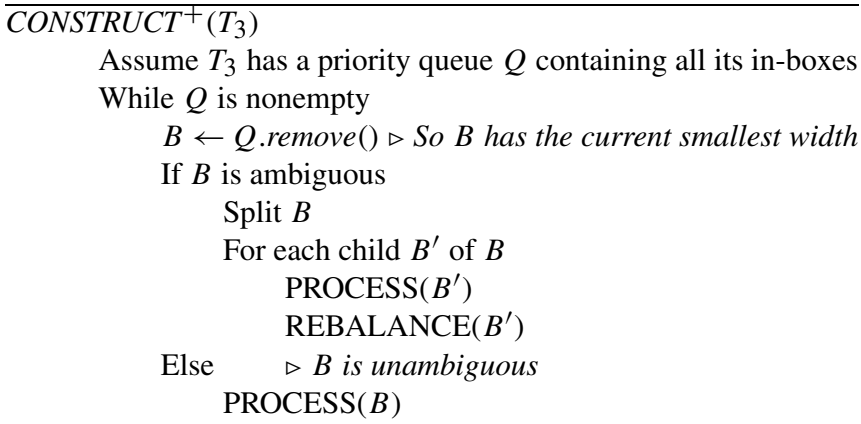

\section{Correctness of Balanced Cxy Algorithm}

The statement is similar to that for the Regular Cxy Algorithm:

Theorem 7 (Correctness of Balanced Cxy Algorithm) The algorithm terminates, provided that $S$ intersects $\partial R\left(T_{0}\right)$ only transversally and $f$ is nonsingular in $R\left(T_{0}\right)$. Moreover, the output graph $G$ is isotopic to $S \cap R\left(T_{0}\right)$.

Let us first prove termination: the termination of the Boundary Phase and Subdivision Phases follows from Lemma 3. But we must also be sure that $\operatorname{CONSTRUCT}^{+}\left(T_{3}\right)$ is terminating because of its splitting of ambiguous boxes and rebalancing. To see that this is a finite process, we observe that when a box $B$ is split in $C O N S T R U C T^{+}$, it is "triggered" by an adjacent box $B^{\prime}$ of smaller width. Thus, the minimum width of boxes in the quadtree is invariant. This implies termination.

The Construction Phase assumes the following property:

Lemma 8 Each in-box has 0, 2 or 4 vertices on its sides. If it has 4 vertices, then two of them will lie on a common side.

We omit the proof which amounts to a case analysis. This is similar to the PV Algorithm [20], but we actually have a new possibility: it is possible to have two vertices on the east and two vertices on the west side of the in-box as shown Fig. 7(e). 
Next, we must show partial correctness. Let us see why the proof for the Regular Cxy Algorithm does not work here: in the key lemma there (Lemma 5), we transform the function $f_{i-1}$ to $f_{i}$ by a reduction step that removes a convergent pair $(a, b)$ that is minimal in the partial order $P\left(f_{i-1}\right)$. Now, there can be "obstructions" to this reduction: in Fig. 4(iii), the pair $\left(a^{\prime}, b^{\prime}\right)$ is an upward convergent of $e^{\prime}$. But in the Balanced Cxy Algorithm, the box $B^{\prime}$ might be split. Say $e^{\prime}$ is thereby split into subsegments $e_{a}^{\prime}$ and $e_{b}^{\prime}$ where $a^{\prime} \in e_{a}^{\prime}$ and $b^{\prime} \in e_{b}^{\prime}$. Thus, $\left(a^{\prime}, b^{\prime}\right)$ is no longer a consecutive pair on any segment, and so $(a, b)$ is now the minimal pair in $P\left(f_{i-1}\right)$. There are two possibilities: (1) We might still be able to reduce the pair $\left(a^{\prime}, b^{\prime}\right)$, but we note that the new $f_{i}$ is no longer consistent with $f_{i-1}$ relative to $T_{3}$. (2) It might also happen that $B^{\prime}$ was split because the component $X_{a}^{\prime}$ of $S \cap B^{\prime}$ with endpoint $a^{\prime}$ and the component $X_{b}^{\prime}$ with endpoint $b^{\prime}$ are different, so we cannot do reduction.

In view of the above discussion, we say that an upward convergent $(a, b) \in P(f)$ is irreducible if it is minimal in the partial order $P(f)$ but it is not an incursion pair (see Fig. 2(iii) and Fig. 7 (e)). The following lemma is critical in the correctness proof. It says that if there exists irreducible minimal pairs, then there exist ambiguous boxes:

Lemma 9 Let $T$ be a balanced quadtree that is compatible with $f$. Let $Q_{u}$ (resp., $Q_{d}$ ) be the set of all minimal upward (downward) convergent pairs of T. Assume that $Q_{u} \cup Q_{d}$ is nonempty and that each pair in $Q_{u} \cup Q_{d}$ is irreducible.

(i) If a segment e contains a convergent pair of $Q_{u}$, then e is the entire south side of an in-box.

(ii) One of the in-boxes of $T$ is ambiguous.

Proof Let $e$ be a segment containing a pair $(a, b) \in Q_{u} \cup Q_{d}$. Without loss of generality, $(a, b)$ is an irreducible upward convergent pair. Assume that $e$ lies in the south side of in-box $B$. See Fig. 4(iii).

(i) First, we show that $e$ is the entire south side of $B$. In other words, the south side of $B$ is not composed of two segments, one of which is $e$. Since $C_{x y}(B)$ holds and there are two distinct points $a, b$ on the south side of $B$, it follows that $0 \notin f_{y}(B)$. As usual, let $X_{a}, X_{b}$ be the connected components of $f^{-1}(0) \cap B$ with one endpoint at $a, b$ (resp.). Clearly, $X_{a} \neq X_{b}$ since $(a, b)$ is irreducible. If the other endpoints of $X_{a}$ and $X_{b}$ are $a^{\prime}$ and $b^{\prime}$ (resp.), then $a^{\prime}$ and $b^{\prime}$ lie on the north side (call it $e^{\prime}$ ) of $B$. Moreover, $a<_{x} a^{\prime}<_{x} b^{\prime}<_{x} b$, and, by irreducibility of $(a, b)$, we must have $a^{\prime}, b^{\prime}$ lying in different subsegments of $e^{\prime}$. Then the subsegment $e_{a}^{\prime}$ containing $a^{\prime}$ (resp., $b^{\prime}$ ) would have $w\left(e_{a}^{\prime}\right) \leq w(e) / 2$. If $e$ is not the entire south side of $B$, then this contradicts the assumption that $T$ is balanced because $w(B) \geq 2 w(e) \geq 4 w\left(e_{a}^{\prime}\right)$.

(Of course, an analogous statement is true if $e$ contains a pair of $Q_{d}$ : in this case, $e$ must be the entire north side of an in-box.)

(ii) We next show that $B$ must be ambiguous under the additional assumption that the width $w(e)$ of $e$ is minimum among all such choices of $e$ (i.e., the minimum-width segment which contains a pair of irreducible convergent pair). We now know that $e$ is the entire south side of $B$ (recall the assumption that $e$ lies in the south side of in-box $B$ and the pair $(a, b)$ is an irreducible upward convergent pair). We will use Fig. 4(iv) 
to illustrate the following arguments. Note that $b$ lies in the right half of $e$ and $a$ lies in the left half of $e$.

First, we show that all the corners of $B$ have the same sign under $f$. Without loss of generality, assume that $f_{y}(B)>0$ and $f((a+b) / 2)<0$. Then we claim that all the corners must be positive.

Suppose that the southeast corner of $B$ is negative. Then $S=f^{-1}(0)$ must intersect $e$ between $b$ and the southeast corner. We may choose $c$ so that $c$ is closest to $b$ among all the intersections. We have that $a<_{x} b<_{x} c$ and $(b, c)$ is a downward convergent pair (since $(a, b)$ is an upward convergent pair). Let $\left(b^{\prime \prime}, c^{\prime \prime}\right)$ be the minimal convergent pair where $(b, c) \succeq\left(b^{\prime \prime}, c^{\prime \prime}\right)$ (note that $\left(b^{\prime \prime}, c^{\prime \prime}\right)$ might be $b$ and $c$ themselves). By assumption, $\left(b^{\prime \prime}, c^{\prime \prime}\right)$ is irreducible. Say $\left(b^{\prime \prime}, c^{\prime \prime}\right)$ lies in a segment $e^{\prime \prime}$. By part (i), we know that $e^{\prime \prime}$ is the complete north side of an in-box $B^{\prime \prime}$. Let $X_{b}^{\prime \prime}, X_{c}^{\prime \prime}$ denote the connected components of $S \cap B^{\prime \prime}$ with endpoints $b^{\prime \prime}, c^{\prime \prime}$ (resp.). By the irreducibility of $\left(b^{\prime \prime}, c^{\prime \prime}\right)$, the other endpoints of $X_{b}^{\prime \prime}$ and $X_{c}^{\prime \prime}$ must lie in separate segments. Since $b$ lies in the right half of $e$ and $b<_{x} b^{\prime \prime}<_{x} c^{\prime \prime}<_{x} c$, this implies $w\left(e^{\prime \prime}\right) \leq w(e) / 2$. This contradicts our choice of $w(e)$ to be minimal.

Thus we may assume that the southwest and southeast corners of $B$ are both positive. But the assumption $f_{y}(B)>0$ implies that the northwest and northeast corners are also positive. Recall that the north side of $B$ is $e^{\prime}$ and that it is split into two subsegments. Thus $B$ is ambiguous iff the midpoint $m\left(e^{\prime}\right)$ of $e^{\prime}$ has negative sign. Note that $a^{\prime}<_{x} m\left(e^{\prime}\right)<_{x} b^{\prime}$. Note that if there are any incursions of the curve $f^{-1}(0)$ into box $B$ between $a^{\prime}$ and $b^{\prime}$, then we would have some $c^{\prime}$ such that either $\left(a^{\prime}, c^{\prime}\right)$ or $\left(c^{\prime}, b^{\prime}\right)$ forms an upward convergent pair. This would contradict the minimality of $(a, b)$. But if there are no incursions between $a^{\prime}$ and $b^{\prime}$, then the sign of $m\left(e^{\prime}\right)$ would be negative (same as $f((a+b) / 2))$. This completes our proof.

As a corollary, if $T$ has no ambiguous boxes, then there can be no convergent pairs $\left(Q_{u} \cup Q_{d}=\emptyset\right)$.

The following is the analogue of Lemma 5 for the Regular Cxy Algorithm:

Lemma 10 Let the quadtree $T$ be balanced and compatible with $f$. If $T$ contains no ambiguous boxes, then the graph $G:=\operatorname{CONSTRUCT} T^{f}(T)$ is isotopic to $f^{-1}(0) \cap$ $R(T)$.

Proof Proceed as in the proof of Lemma 5: we can repeatedly reduce each minimal convergent pair (upward, downward, left, or right) by transforming $f_{0}=f$ to $f_{1}, f_{2}, \ldots$. Let $\bar{f}$ be the final function when we cannot further reduce any minimal pair. According to Lemma 9, this means that there are no more convergent pairs (otherwise, there would be ambiguous boxes). This means that the curve $\bar{S}=\bar{f}^{-1}(0)$ must intersect each segment $e$ at most once. We conclude that $G=\operatorname{CONSTRUCT}^{f}(T)$ is isotopic to $\bar{S} \cap R(T)$.

\section{Conclusion of the Correctness Proof}

Proof The quadtree $T_{3}$ is balanced and compatible with $f$. When we invoke CONSTRUCT ${ }^{+}\left(T_{3}\right), T_{3}$ is further transformed by splits of ambiguous boxes and their 


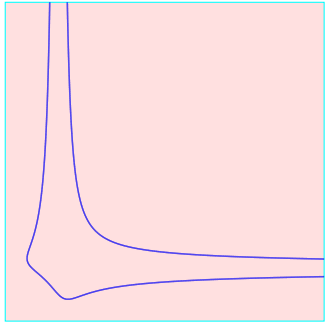

(a) Original Curve

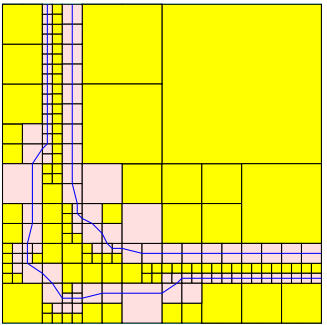

(b) PV

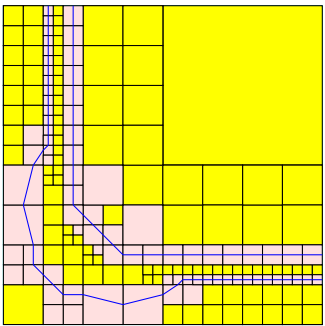

(d) Balanced Cxy

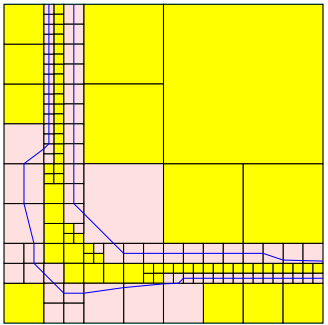

(c) Snyder

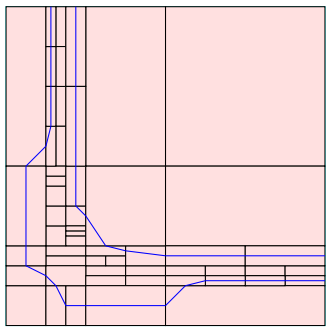

(e) Rectangular Cxy

Fig. 8 Approximation of $f(X, Y)=X^{2} Y^{2}-X+Y-1=0$ inside the box $[(-2,-10),(10,2)]$ using $\mathrm{PV}$, Snyder, Cxy, and Rect

rebalancing. Let $T_{4}$ be the final quadtree. It is clear that the output of $C O N S T R U C T^{+}$ on $T_{3}$ is the same as what the original CONSTRUCT would produce on input $T_{4}$ :

$$
\operatorname{CONSTRUCT}^{+}\left(T_{3}\right)=\operatorname{CONSTRUCT}\left(T_{4}\right) .
$$

Clearly, $f$ is still compatible with $T_{4}$. By Lemma 10, the straightline graph $G=$ CONSTRUCT $\left(T_{4}\right)$ is isotopic to $f^{-1}(0) \cap R(T)$. This concludes our proof.

\section{Rectangular Cxy Algorithm}

The recent meshing algorithms [6, 20, 27] all assume full-splits (subdividing a box into four subboxes). We now introduce an Cxy algorithm that can do half-splits. The boxes are no longer squares, and hence the next algorithm is known as the Rectangular Cxy Algorithm. This algorithm is even more adaptive than the Balanced Cxy Algorithm, and this can be illustrated with the curve $X^{2} Y^{2}-X+Y=1$ shown in Fig. 8. The curve has preferred directions in the horizontal and vertical directions. Our algorithm can automatically produce rectangles that are elongated along the corresponding directions to adapt to the curve-see Fig. 8(e). As a result, the number of subdivisions can be drastically reduced as compared to algorithms based on square boxes. The new algorithm differs from balanced Cxy in three major aspects:

First, we need an arbitrary but fixed parameter $r$ called the aspect ratio bound. For a box $B$, let $\alpha(B):=w_{y}(B) / w_{x}(B)$. Then its aspect ratio is defined as $\rho(B):=$ 
$\max \left\{\alpha(B), \frac{1}{\alpha(B)}\right\} \geq 1$. We require that all boxes in our quadtree satisfy $\rho(B) \leq r$. This ensures the termination of our algorithm.

Second, we modify the Subdivision Phase as follows: For each in-box $B$ in the queue, we must decide how to tag it, or how to split and tag its children. This is accomplished by a new splitting procedure, which amounts to checking the following three lists of conditions (in this order):

$$
\left.\begin{array}{ll}
L_{0}: & C_{0}(B), C_{x y}(B) \\
L_{\text {out }}: & C_{0}\left(B_{12}\right), C_{0}\left(B_{34}\right), C_{0}\left(B_{14}\right), C_{0}\left(B_{23}\right) \\
L_{\text {in }}: & C_{x y}\left(B_{12}\right), C_{x y}\left(B_{34}\right), C_{x y}\left(B_{14}\right), C_{x y}\left(B_{23}\right)
\end{array}\right\}
$$

We stop at the first verified condition. If a condition in $L_{0}$ is verified, we tag $B$ as an in- or out-box, accordingly. If a condition in $L_{\text {out }}$ or $L_{\text {in }}$ is verified, we do a half-split of $B$ to produce the child that satisfies that condition. That child is tagged as out (if an $L_{\text {out }}$ condition) or in (if an $L_{\text {in }}$ condition). The other child is pushed back into the queue. Finally, if no condition is verified, we do a full-split and push the four children into the queue.

Actually, this splitting procedure must be slightly modified in order to respect the aspect ratio bound (this amounts to avoid testing the first half of the conditions in $L_{\text {out }}$ and $L_{\text {in }}$ if $\alpha(B)<2 / r$, and to avoid testing the second half if $\alpha(B)>r / 2$. Note that there is considerable opportunity for sharing, and thus optimization, when implementing the arithmetic operations to check the 10 conditions of (8).

Third, we must track the "splitting depth" of a node in the quadtree by a pair of natural numbers, called its $x$-depth and $y$-depth. These count the number of vertical and (respectively) horizontal splits from the root to the given node. A full-split counts both a vertical and a horizontal split. We now say that a box $B$ is $x$-balanced if its north and south neighbors have $x$-depth at most 1 away from the $x$-depth of $B$; similarly for $y$-balanced with respect to its east and west neighbors. The Balancing Phase is easily modified to only doing half-splits in order to achieve the balance condition for all boxes. One strategy is to first achieve $x$-balance for all in-boxes, then to do the same for $y$-balance. Finally, in the Construction Phase, we modify $\operatorname{CONSTRUCT}^{+}\left(T_{3}\right)$ so that ambiguity-based priority queue should distinguish between an $y$-ambiguity (e.g., Fig. 6(a)-(c')) that must be resolved by a horizontal split, or a $x$-ambiguity that requires a vertical split.

\section{Ensuring Geometric Accuracy}

So far, we have focused on computing the correct isotopy. We now consider the process of refinement whose goal is geometric accuracy, i.e., to ensure an approximation $G$ that is $\varepsilon$-close to $S \cap B_{0}$. The "small normal variation" $C_{1}$ predicate is quite strong, so that it is quite easy to use for refinement in the PV algorithm (this is implicit in $[19,20])$. To see this explicitly, we claim that it suffices to ensure that for any in-box $B$, if it has at least one edge of $G=(V, E)$, then its diameter is $\leq \varepsilon / 4$. Then any neighbor $B^{\prime}$ of $B$ has diameter at most $\varepsilon / 2$. Thus, each edge $e$ in $B$ is isotopic to a curve component $X$ of $S \cap\left(B \cup B^{\prime}\right)$. But the distance between any two points in $B \cup B^{\prime}$ is $\leq \varepsilon \sqrt{(1 / 2)^{2}+(3 / 4)^{2}}<\varepsilon$. With our $C_{x y}$ predicate, no such bound on 
Fig. 9 Halfcircle argument

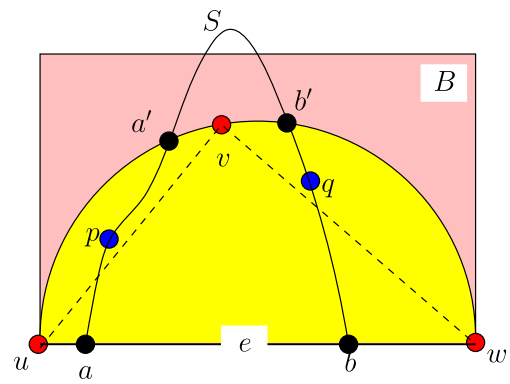

geometric accuracy is possible because our curve could now escape arbitrarily far away from our constructed approximation via undetected excursions. Below, we develop a generalization of the $C_{1}$ predicate to capture geometric accuracy bounds for rectangular boxes.

\subsection{Extending the Buffer Lemma of Plantinga and Vegter}

It is noted in Plantinga and Vegter that if $B$ is a square box, and $C_{1}(B)$ holds, then any "incursion" of the curve $S$ along a side of $B$ cannot leave $B$. Thus, $B$ acts as a "buffer" area within which any isotopic variation of the curve $S$ must lie. Their result is still true if $B$ is "almost square," as captured by our next lemma:

Lemma 11 (Buffer Property) Let $(a, b)$ be a convergent pair relative to box $B$. Without loss of generality, assume that $(a, b)$ lies on the south side e of $B$. Let $X_{a}$ and $X_{b}$ (resp.) be the connected components of $S \cap B$ with one endpoint at a and $b$ (resp.) If condition $C_{1}(B)$ holds and $\alpha(B) \geq 1 / 2$, then $X_{a}=X_{b}$.

Proof Figure 9 illustrates our proof. Let $H$ be the upper halfcircle with diameter $e$. Since $\alpha(B) \geq 1 / 2, H$ must lie completely inside the rectangle $B$. If $X_{a} \neq X_{b}$, then the component $X_{a}$ must leave the interior of the halfcircle $H$ at some first point $a^{\prime} \in H$; similarly, $X_{b}$ must leave at some point $b^{\prime} \in H$. By the mean value theorem, there is a point $p$ (resp., $q$ ) on $X_{a}$ (resp., $X_{b}$ ) whose slope is equal to the slope of the segment $\left[a, a^{\prime}\right]$ (resp., $\left[b, b^{\prime}\right]$ ). Let the endpoints of the side $e$ be $u, w$ and pick any point $v \in H$ between $a^{\prime}$ and $b^{\prime}$. Clearly, the slope at $p$ is more than the slope of $[u, v]$, and the slope at $q$ is more negative than the slope of $[v, w]$. Thus, the angle between the normals at $p$ and $q$ must be greater than the angle between the two normals of the segments $[u, v]$ and $[v, w]$. But the latter angle is exactly $90^{\circ}$ (since $H$ is a halfcircle). This contradicts the fact that $C_{1}(B)$ holds.

We further loose the constraint on $B$ from "almost square" to a rectangle with arbitrary aspect ratio $\alpha(B)$. We also need to do some change on the $C_{1}$ predicate.

\subsection{Generalized $C_{1}$ Predicate}

We now generalize the $C_{1}$ predicate of Plantinga and Vegter so that it guarantees the same buffering effect for any rectangle, not just those with aspect ratio $\leq 2$. 
For any box $B$, define the linear map

$$
T_{B}: \mathbb{R}^{2} \rightarrow \mathbb{R}
$$

where $T_{B}(x, y):=(x, y / \alpha(B))$. Note that $B^{\prime}=T_{B}(B)$ is a square. Alternatively, the inverse of $T_{B}$ is $T_{B}^{-1}(x, y)=(x, \alpha(B) y)$. For any function $f: \mathbb{R}^{2} \rightarrow \mathbb{R}$, define

$$
f^{B}: \mathbb{R}^{2} \rightarrow \mathbb{R}
$$

where $f^{B}(p)=f\left(T_{B}^{-1}(p)\right)$. It is easy to see that

$$
f^{B}\left(T_{B}(p)\right)=f\left(T_{B}^{-1}\left(T_{B}(p)\right)\right)=f(p)
$$

and hence $f^{B}\left(B^{\prime}\right)=f(B)$. Let $C_{1}^{*}$ denote the "generalized $C_{1}$ predicate" which holds at a box $B$, provided that

$$
C_{1}^{*}(B): 0 \notin\left(\square f_{x}^{B}\left(B^{\prime}\right)\right)^{2}+\left(\square f_{y}^{B}\left(B^{\prime}\right)\right)^{2} .
$$

We have the following:

Lemma 12 Let $(a, b)$ be an upward convergent pair of a segment $e$, where $e$ is the south side of a box $B$. Let $X_{a}$ and $X_{b}$ (resp.) be the connected components of $f^{-1}(0) \cap B$ with one endpoint at $a$ and $b$ (resp.) If condition $C_{1}^{*}(B)$ holds, then $X_{a}=X_{b}$ (i.e., $X_{a}$ is a B-intrusion).

Proof Note that $C_{1}^{*}(B)$ means that $C_{1}^{g}\left(B^{\prime}\right)$ holds, where $g=f^{B}$ (see the superscript notation for $C_{1}^{g}\left(B^{\prime}\right)$ in Sect. 4.2). Let $X_{T_{B}(a)}$ and $X_{T_{B}(b)}$ be the connected components of $g^{-1}(0) \cap B^{\prime}$ with one endpoint at $T_{B}(a)$ and $T_{B}(b)$ (resp.). From the previous lemma we know that $X_{T_{B}(a)}=X_{T_{B}(b)}=X^{\prime}$ and that $X^{\prime}$ is completely included inside $B^{\prime}$. Since $T_{B}$ is a bijection that maps $B^{\prime}$ to $B$, we can conclude that $X=T_{B}^{-1}\left(X^{\prime}\right)=T_{B}^{-1}\left(X_{T_{B}(a)}\right)=T_{B}^{-1}\left(X_{T_{B}(b)}\right)$ is completely included inside $B$, i.e., $X_{a}=X_{b}$.

\subsection{Refinement based on the Generalized $C_{1}$ Predicate}

We introduce the concept of safety of segments. Intuitively, a segment $s$ is safe if there can be no incursion or excursion along $s$.

Let $T_{3}$ be a quadtree from the Subdivision Phase of our Rectangular Cxy Algorithm. For each (rectangular) box $B$ in $T_{3}$, we will classify some of its sides as safe relative to $B$ :

- If $C_{0}(B)$ holds, then each of its sides is safe relative to $B$.

- If $C_{x}(B)$ holds, then its north and south sides are safe relative to $B$. Similarly, if $C_{y}(B)$ holds, then its east and west sides are safe.

More generally, a segment $s$ is safe (not relative to any box) if there exists $s^{\prime}$ such that $s \subseteq s^{\prime}$ and $s^{\prime}$ is safe relative to some box $B^{\prime}$. It is easy to see that we can effectively know whether a segment $s$ is safe from the information derived in 
constructing the tree $T_{3}$. In particular, when we determine that a box satisfies $C_{x y}$, we actually know whether it satisfies $C_{x}$ or $C_{y}$ (or even both).

The safety of some (but not all) segments can be deduced by looking at the presence of vertices along the sides of a box. For instance, in Fig. 7(a)-(f), we have indicated by thick edges those sides that we know to be safe because of the presence of vertices. Note that we do not have any thick edges for Case (a) even though we know at least two of them must be safe. In Case (f), we can also deduce that the eastern side to be "safe," not according to our definition above, but in the extended sense that no incursion or excursion can occur. We could, but need not, exploit such extended notions of safety.

\subsection{Exploiting Safe Segments for Refinement}

Lemma 13 Let $s$ be a safe segment.

(i) Then the curve $S=f^{-1}(0)$ intersects $s$ at most once, i.e., $|S \cap s| \leq 1$.

(ii) $|S \cap s|=1$ iff $f$ have different signs at the endpoints of $s$.

Proof (i) If $s$ is safe, then $s \subseteq s^{\prime}$ where $s^{\prime}$ is safe relative to some box $B^{\prime}$. If $C_{0}\left(B^{\prime}\right)$ holds, then clearly $|S \cap s|=0$. If $C_{x y}\left(B^{\prime}\right)$ holds such that $S$ is parameterizable along the direction of $s$, then clearly $|S \cap s| \leq 1$.

(ii) If $f$ has different signs at the endpoints of $e$, then $|S \cap e|$ is odd. By part (i), $|S \cap e|=1$. Conversely, if $f$ have the same sign at the endpoints of $e$, then $|S \cap e|$ is even. By part (i), $|S \cap e|=0$.

Let $s$ be a segment. We say that $s$ is soft if it is not safe. Suppose that $B$ is a terminal box (i.e., satisfies $C_{x y}$ but not $C_{0}$ ) with at least one soft side. Then the distance from this soft side to the opposite side is called the soft distance of $B$. Note that this soft distance is uniquely defined. If $B$ has no soft side, then the soft distance is 0 by definition. If the soft distance is $d$, then any incursion into $B$ can be removed by modifying the curve within a Hausdorff distance of $d$.

There are three kinds of curve component $C=B \cap S$ in box $B$ as illustrated in Fig. 2: incursion, cut or corner components. We consider bounds on the dimension of $B$ in order that our straightline approximations to $C$ is within Hausdorff distance $\varepsilon / 2$ from $C$.

(a) Suppose that $C$ is an incursion, i.e., both endpoints of $C$ lie on one side of $B$. If $B$ has soft distance at most $\varepsilon / 2$, then, as noted, $C$ can be removed by perturbing the curve by a Hausdorff distance of $\varepsilon / 2$.

(b) Suppose that $C$ is a cut component, i.e., the endpoints of $C$ lie on opposite sides of $B$. If $s$ is a side of $B$ containing an endpoint of $C$, then we want the length of $s$ to be at most $\varepsilon$. This ensures that our linear approximation is within Hausdorff distance $\varepsilon / 2$ from an actual curve component within $B$.

(c) Suppose that $C$ is a corner component, i.e., the endpoints of $C$ lie on adjacent sides of $B$. In this case, we want each side of $B$ to have length at most $\sqrt{2} \varepsilon / 3$. Again, it ensures that our straightline approximation is within Hausdorff distance $\varepsilon / 2$ from an actual curve component within $B$. 
We now sketch how to incorporate $\varepsilon$-refinement into the Rectangular Cxy Algorithm. The idea is to ensure that each terminal box has dimensions bounded as in (a)-(c) above. It is easiest to assume that the original subdivision phase has been carried out (so all boxes are known to satisfy $C_{0}$ or $C_{x y}$ ). We make another pass through the list of boxes that satisfies $C_{x y}$ but not $C_{0}$. Such a box $B$ is passive if the function $f$ has uniform signs (either all positive or all negative) at the corners on the boundary of $B$; otherwise it is active. Note that the approximate curve $G$ passes through $B$ iff $B$ is active. We keep $B$ if the following conditions hold:

$\left(\mathrm{a}^{\prime}\right)$ If $B$ is passive and has at least one soft side, then we check that the generalized predicate $C_{1}^{*}(B)$ holds. Under this condition, any undetected entry of the curve into $B$ must represent an incursion. We require the soft distance of $B$ to be at most $\varepsilon / 2$.

$\left(b^{\prime}\right)$ If $B$ is active and has sign changes on two opposite sides, then we require the lengths of these sides to be at most $\varepsilon / 2$.

$\left(\mathrm{c}^{\prime}\right)$ If $B$ is active and has sign changes on two adjacent sides, then we require the lengths of all sides to be at most $\sqrt{2} \varepsilon / 3$.

If any of the above conditions fail, we split $B$ and put any child that fails the $C_{0}$ predicate back into the queue. This completes our description of the modified subdivision phase. Other phases are unchanged. The correctness follows easily from our discussion.

The above refinement method can also be adapted for the Balanced Cxy algorithm. Here we only have square boxes. It amounts to ensuring that each passive box $B$ with at least one soft side also has width at most $\varepsilon / 2$ and satisfies $C_{1}$ and that each active box $B$ has width at most $\sqrt{2} \varepsilon / 3$.

\section{Summary of Experimental Results}

We report on our experimental results. Our code is developed in Java on the Eclipse Platform (SDK Version 3.3.0). The hardware is Dell Laptop Inspiron 6400, with Intel Core2 Duo Mobile Processor T2500 (2.0 GHz, 667 FSB, 2MB shared L2 Cache) and $2.0 \mathrm{~Gb}$ of RAM. We use the default Java heap memory $256 \mathrm{MB}$ (some runs result in OutOfMemoryError). Note that this implementation is based on machine arithmetic. But since all arithmetic operations use only ring operations and divide by 2 , there are no round-off errors except for under/overflows. Our examples below do not reach such limits. The current code is available on http://cs.nyu.edu/exact/papers/. The code has been translated into $\mathrm{C}++$ for distribution with our open source Core Library. We implemented four algorithms: PV, Snyder, Balanced Cxy, and Rectangular Cxy. For Snyder's algorithm, the boundary root isolation is carried out using the 1D analogue, namely the EVAL algorithm (see [7, 8, 17]). For brevity, the Balanced Cxy Algorithm and Rectangular Cxy Algorithm will be known as Cxy and Rect, respectively.

We have not yet implemented two concepts discussed in this paper: Boundary processing for arbitrary input geometry $R\left(T_{0}\right)$ (Sect. 3) and exploiting safe segments for geometric accuracy (Sect. 8). As stated in our introduction, most of our experiments 


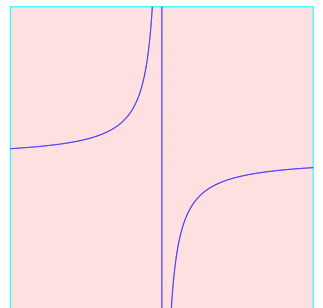

(a) Original Curve

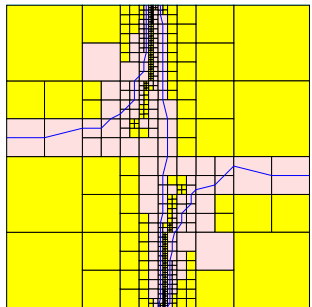

(b) PV

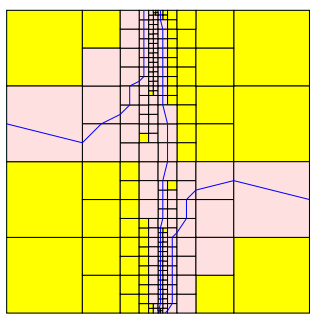

(d) Balanced Cxy

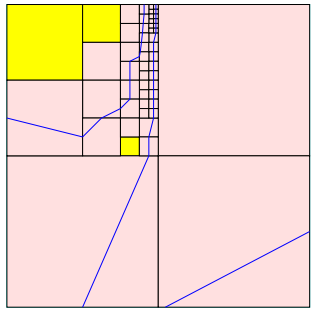

(c) Snyder

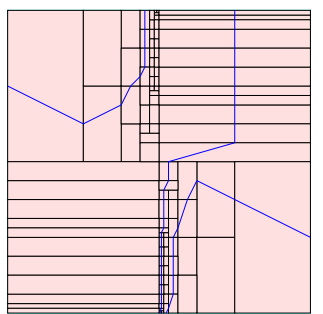

(e) Rectangular Cxy

Fig. 10 Approximation of $f(X, Y)=X(X Y-1)=0$ inside the box $[(-4,-4),(4,4)]$. (b), (d), (e) are from PV, Cxy, and Rect, inside the box $[(-3.9,-3.9),(4.1,4.1)],(\mathbf{c})$ is from Snyder

are concerned computing the correct isotopy, ignoring geometric accuracy. But we could easily and cheaply improve geometric accuracy in our approximation graphs by using interpolation: instead of choosing vertices at midpoints of segments, we choose some linearly interpolated point.

We now summarize our main conclusions, based on compare four algorithms: Cxy, Rect, PV, and Snyder. We also briefly compare to EXACUS from the Max-Planck Institute of Computer Science.

(1) Cxy can be significantly faster than $P V$ and Snyder. Figure 1 is gotten by running these algorithms on the curve $f(X, Y)=X^{2}(1-X)(1+X)-Y^{2}+0.01=0$ inside the box $[(-1.5,-1.5),(1.5,1.5)]$. This example is from [20]. Cxy is twice as fast as PV and Snyder, and Rect is the fastest: the PV produces 196 boxes in 31 milliseconds, Snyder produces 112 boxes in 37 milliseconds, Cxy produces 112 boxes in 16 milliseconds, and Rect produces 76 boxes in 15 milliseconds.

(2) When we add refinement, the improvement is minimal. We currently use a simplistic approach based on the $C_{1}$ predicate. We believe this part can be sped up, for example, by implementing the method from Sect. 8. The refined curve, with precision $\epsilon=0.005$, is shown in Fig. 1(a). PV produces 8509 boxes in 219 ms, while Cxy produces 8497 boxes in $204 \mathrm{~ms}$.

(3) Rect can be significantly faster than Cxy. For example, let the aspect ratio bound be $r=5$. Running the algorithms on the curve $f(X, Y)=X(X Y-1)=0$ in the box $B_{s}:=[(-s,-s),(s, s)]$ (Figs. 10 (b), (c), (d), and (e) show the cases where $s=4$. Snyder will not terminate when the curve intersects the sides of the boxes tangentially, so we shift the initial box a little bit). We get the Table 1 (OME = OutOfMemoryError): 
Table 1 Rect $>$ Cxy $>$ PV

\begin{tabular}{llll}
\hline \#Boxes/Time $(\mathrm{ms})$ & $s=15$ & $s=60$ & $s=100$ \\
\hline PV & $5686 / 157$ & OME & OME \\
Cxy & $2878 / 125$ & $45790 / 2750$ & OME \\
Rect & $288 / 31$ & $4470 / 609$ & $13042 / 4266$ \\
\hline
\end{tabular}

Fig. 11 Approximation of $f(X, Y)=X(X Y-1)=0$ inside the box

$[(-15,-15),(15,15)]$ using Rect with maximum aspect ratios of $10,20,40$, and 80

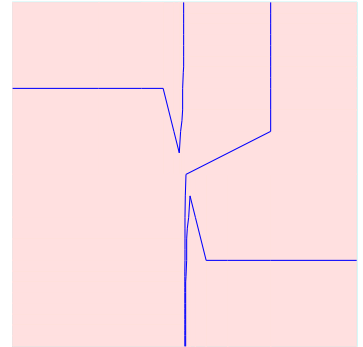

(a) $r=10$

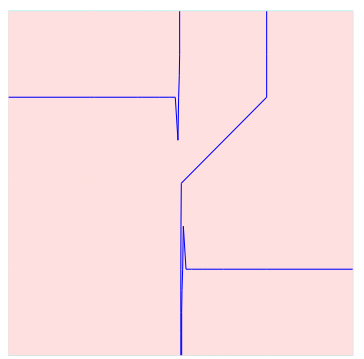

(c) $r=40$

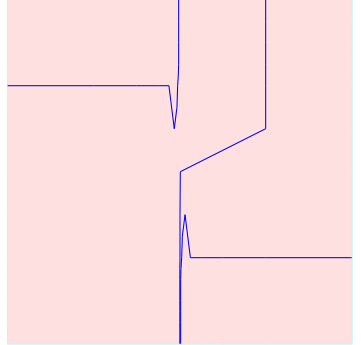

(b) $r=20$

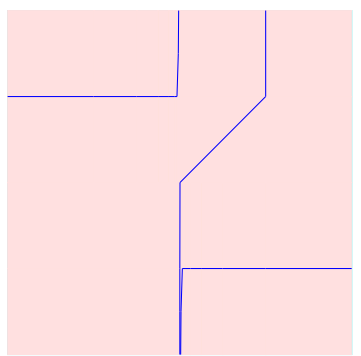

(d) $r=80$

Table 2 Rect can exploit larger aspect ratio

\begin{tabular}{llll}
\hline \#Boxes/Time (ms) & $s=15$ & $s=60$ & $s=100$ \\
\hline$r=10$ & $150 / 16$ & $2242 / 265$ & $6540 / 1109$ \\
$r=20$ & $82 / 15$ & $1134 / 109$ & $3282 / 406$ \\
$r=40$ & $48 / 15$ & $574 / 62$ & $1656 / 172$ \\
$r=80$ & $32 / 0$ & $296 / 32$ & $842 / 78$ \\
\hline
\end{tabular}

(4) Increasing the aspect ratio bounds can speed up the performance of Rect. Using the same curve and box as before, we now look at the performance of Rectangular Cxy with variable aspect ratio bounds of $r=10,20,40,80$. Figure 11 shows the case where $r=15$. Table 2 shows a proportional speedup (time $=0$ means time $<1 \mathrm{~ms}$ ):

(5) Sometimes Snyder is faster than Balanced Cxy. We now show an example in which Cxy is slower than Snyder; in turn, Snyder is slower than Rect. When we want to ensure geometric closeness, it is clear that our new approach is considerably faster because Snyder is not forced to subdivide the terminal boxes until their diameters are $\leq \varepsilon$. We compare PV, Cxy, Rect (with maximum aspect ratio $r=257$ ) and Snyder on 


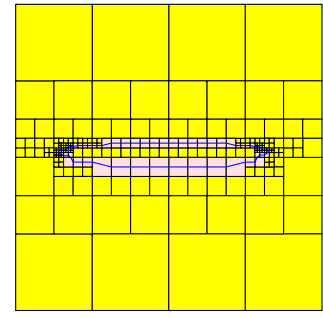

(b) PV

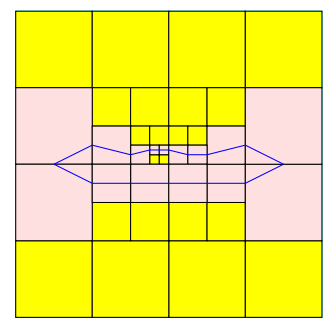

(d) Balanced Cxy

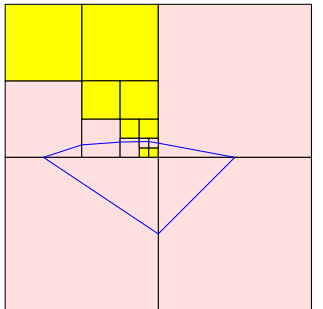

(c) Snyder

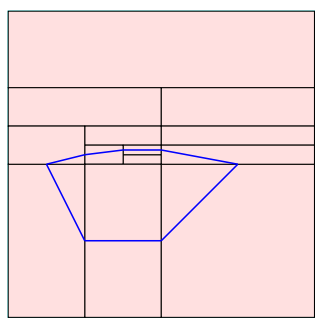

(e) Rectangular Cxy

Fig. 12 Approximation of $f(X, Y)=X^{2}+100 Y^{2}-1=0$ in the box $[(-1.4,-1.4),(1.5,1.5)]$ using PV, Snyder, Cxy, and Rect

Table 3 Rect $>$ Snyder $>$ Cxy > PV

\begin{tabular}{lllll}
\hline \#Boxes/Time $(\mathrm{ms})$ & $n=4$ & $n=5$ & $n=6$ & $n=7$ \\
\hline PV & $1825 / 62$ & $6415 / 234$ & $20806 / 1219$ & $65926 / 9219$ \\
Snyder & $25 / 16$ & $31 / 16$ & $34 / 31$ & $40 / 31$ \\
Cxy & $175 / 15$ & $769 / 218$ & $694 / 172$ & $754 / 172$ \\
Rect & $17 / 0$ & $14 / 0$ & $25 / 0$ & $29 / 0$ \\
\hline
\end{tabular}

the curve $f(X, Y)=X^{2}+a Y^{2}-1=0$ in the box $[(-1.4,-1.4),(1.5,1.5)]$, where $a=10^{n}$ for $n=4, \ldots, 7$ (Fig. 12 shows the cases where $n=2$, Table 3 ).

The curve here is a thin and long oval, so the size of the smallest box would be very small. Both Cxy and PV need to do balancing and produce more boxes than Snyder, so they are more time consuming (note that Cxy is significantly ( $>50$ times) faster than PV when $n=7$ ). Rect produces even fewer boxes than Snyder, and Snyder needs to do root isolation; so it is not surprising that Rect is much faster than Snyder.

(6) In general, Cxy and Rect have better performance than Snyder. We ran Snyder on the curve $f(X, Y)=X(X Y-1)=0$. Since Snyder will not terminate when the curve intersects the sides of the boxes tangentially, we cannot run this example on the box $B_{s}:=[(-s,-s),(s, s)]$. Instead, we chose the initial box to be $B_{n}:=[(-14 \times$ $\left.\left.10^{n},-14 \times 10^{n}\right),\left(-15 \times 10^{n},-15 \times 10^{n}\right)\right]$, where $n=(-1,0,1)$. Figure $10(\mathrm{c})$ shows the case where $B_{0}:=[(-3.9,-3.9),(4.1,4.1)]$. We also tested PV, Cxy, and Rect (with maximum aspect ratio $r=257$ ), see Table 4 . 
Table 4 Rect $>$ Cxy $>$ Snyder $>$ PV

\begin{tabular}{llll}
\hline \#Boxes/Time (ms) & $n=-1$ & $n=0$ & $n=1$ \\
\hline PV & $73 / 0$ & $4417 / 516$ & OME \\
Snyder & $10 / 15$ & $1306 / 125$ & OME \\
Cxy & $13 / 0$ & $1510 / 62$ & OME \\
Rect & $6 / 0$ & $13 / 0$ & $255 / 31$ \\
\hline
\end{tabular}

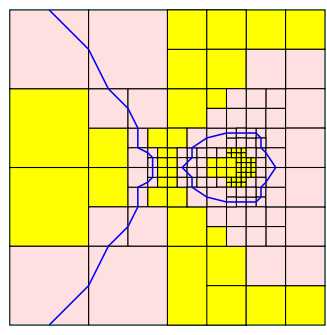

(b) PV

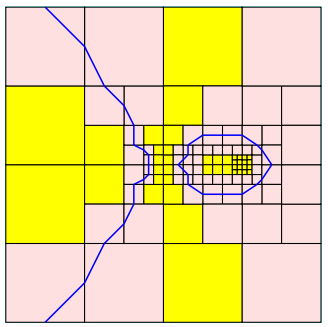

(d) Balanced Cxy

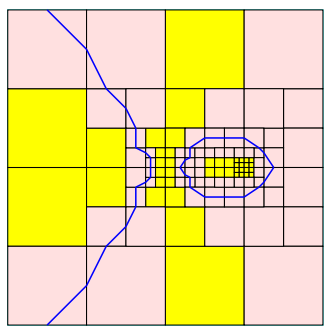

(c) Snyder

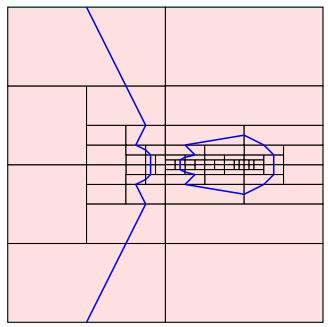

(e) Rectangular Cxy

Fig. 13 Approximation of $f(X, Y)=Y^{2}-X^{2}+X^{3}+0.02=0$ inside the box $[(-1.5,-1.5),(1.5,1.5)]$ using PV, Snyder, Cxy, and Rect

(7) Cxy can work with high-degree curves and sometimes improve on EXACUS. The EXACUS system has a nice web interface accessible from http://exacus.mpi-inf. mpg.de/cgi-bin/xalci.cgi. EXACUS is based on strong algebraic methods and can handle singularities. The following examples show that our algorithm could be much faster than EXACUS.

- Approximating the curve $f(X, Y)=X^{100}+Y^{100}-1=0$ in the box $B_{0}:=$ $[(-2,-2),(2,2)]$ : Cxy takes 9.451 seconds, while EXACUS is timed out.

- Approximating the curve: $f(X, Y)=\left(X^{2}+Y^{2}\right)^{k}-4 X^{2} Y^{2}-0.01=0$ inside the box $B_{0}:=[(-1,-1),(1,1)]$, EXACUS is timed out when $k \geq 7$. Cxy takes 14.235 seconds when $k=7,22.123$ seconds when $k=8,27.607$ seconds when $k=9$, and $<3$ minutes when $k=10$.

(8) Two more examples. We had already seen Fig. 8 for the curve $f(X, Y)=$ $X^{2} Y^{2}-X+Y-1=0$ inside the box $[(-2,-10),(10,2)]$. PV produces 211 boxes in 16 milliseconds, Snyder produces 139 boxes in 31 milliseconds, Cxy produces 181 
boxes in 15 milliseconds, and Rect produces 54 boxes in $<1$ millisecond. Another example in Fig. 13 shows the approximation of $f(X, Y)=Y^{2}-X^{2}+X^{3}+0.02=0$ inside the box $[(-1.5,-1.5),(1.5,1.5)]$. PV produces 154 boxes in 15 milliseconds, Snyder produces 106 boxes in 31 milliseconds, Cxy produces 106 boxes in 15 milliseconds, and Rect produces 74 boxes in 15 milliseconds.

\section{Conclusion}

This paper introduces a new algorithm for isotopic approximation of implicit curves that is provably correct, simple, efficient, and easy to implement exactly. The basic idea is to exploit parameterizability (like Snyder) and nonlocal isotopy (like Plantinga and Vegter). These ideas are extended to subdivision boxes of bounded aspect ratio. Our experimental results which compare four algorithms (Plantinga and Vegter, Snyder, Balanced Cxy, and Rectangular Cxy) show that our Rect Cxy Algorithm is the best in all tests and often exhibits great speedup. Future work includes extensions to higher dimensions, implementation of irregular geometries, and exploration of faster techniques for curve refinement. Note that there is no known extension of the PV algorithm to dimension $\geq 4$. Another challenge is to produce efficient practical algorithms for higher dimensions. Galehouse [13] recently provided a new subdivision algorithm for meshing surfaces in any dimension and implemented it in 4 dimensions.

\section{References}

1. Basu, S., Pollack, R., Roy, M.-F.: Algorithms in real algebraic geometry. In: Algorithms and Computation in Mathematics. Springer, Berlin (2003)

2. Boissonnat, J.-D., Cohen-Steiner, D., Mourrain, B., Rote, G., Vegter, G.: Meshing of surfaces. In: Boissonnat and Teillaud [5], Chapter 5

3. Boissonnat, J.-D., Cohen-Steiner, D., Vegter, G.: Isotopic implicit surfaces meshing. In: ACM Symp. on Theory of Computing, pp. 301-309 (2004)

4. Boissonnat, J.-D., Oudot, S.: Provably good sampling and meshing of surfaces. Graph. Models 67(5), 405-451 (2005)

5. Boissonnat, J.-D., Teillaud, M. (eds.): Effective Computational Geometry for Curves and Surfaces. Springer, Berlin (2006)

6. Burr, M., Choi, S., Galehouse, B., Yap, C.: Complete subdivision algorithms, II: Isotopic meshing of singular algebraic curves. In: Proc. Intern. Symp. Symbolic and Algebraic Computation (ISSAC'08), Hagenberg, Austria, 20-23 Jul, 2008, pp. 87-94 (2008).

7. Burr, M., Krahmer, F., Yap, C.: Continuous amortization: A non-probabilistic adaptive analysis technique. In: Electronic Colloquium on Computational Complexity (ECCC), TR09(136), Dec. 2009 (2009)

8. Burr, M., Sharma, V., Yap, C.: Evaluation-based root isolation, Feb. 2009. In preparation

9. Cheng, J.-S., Gao, X.-S., Yap, C.-K.: Complete numerical isolation of real zeros in zero-dimensional triangular systems. J. Symb. Comput. 44(7), 768-785 (2009). Special Issue of JSC based on ISSAC 2007. Available online at JSC

10. Cheng, S.-W., Dey, T., Ramos, E., Ray, T.: Sampling and meshing a surface with guaranteed topology and geometry. In: Proc. 20th ACM Symp. on Comp. Geometry, pp. 280-289 (2004)

11. Eigenwillig, A.: Real root isolation for exact and approximate polynomials using descartes' rule of signs. Ph.D. thesis, University of Saarland, Saarbruecken, Germany, May 2008

12. Eigenwillig, A., Kettner, L., Schmer, E., Wolpert, N.: Complete exact, and efficient computations with cubic curves. In: 20th ACM Symp. on Comp. Geometry, Brooklyn, New York, USA, 08-11 Jun, pp. 409-418 (2004). 
13. Galehouse, B.: Topologically accurate meshing using spatial subdivision techniques. Ph.D. thesis, New York University, Department of Mathematics, Courant Institute, May 2009. From http://cs.nyu.edu/exact/doc/

14. Hong, H.: An efficient method for analyzing the topology of plane real algebraic curves. Math. Comput. Simul. 42, 571-582 (1996)

15. Lorensen, W.E., Cline, H.E.: Marching cubes: A high resolution 3D surface construction algorithm. In: Stone, M.C. (ed.) Computer Graphics (SIGGRAPH '87 Proceedings), vol. 21, July 1987, pp. 163169 (1987)

16. Martin, R., Shou, H., Voiculescu, I., Bowyer, A., Wang, G.: Comparison of interval methods for plotting algebraic curves. Comput. Aided Geom. Des. 19(7), 553-587 (2002)

17. Mitchell, D.P.: Robust ray intersection with interval arithmetic. In: Graphics Interface'90, pp. 68-74 (1990)

18. Moore, R.E.: Interval Analysis. Prentice Hall, Englewood Cliffs (1966)

19. Plantinga, S.: Certified algorithms for implicit surfaces. Ph.D. thesis, Groningen University, Institute for Mathematics and Computing Science, Groningen, Netherlands, Dec. 2006

20. Plantinga, S., Vegter, G.: Isotopic approximation of implicit curves and surfaces. In: Proc. Eurographics Symposium on Geometry Processing, pp. 245-254. ACM Press, New York (2004)

21. Preparata, F.P., Shamos, M.I.: Computational Geometry. Springer, Berlin (1985)

22. Ratschek, H., Rokne, J.: Computer Methods for the Range of Functions. Horwood Publishing, Chichester (1984)

23. Ratschek, H., Rokne, J.G.: SCCI-hybrid methods for 2 d curve tracing. Int. J. Image Graph. 5(3), 447-480 (2005)

24. Schoemer, E., Wolpert, N.: An exact and efficient approach for computing a cell in an arrangement of quadrics. Comput. Geom. 33, 65-97 (2006)

25. Seidel, R., Wolpert, N.: On the exact computation of the topology of real algebraic curves. In: Proc. 21st ACM Symp. on Comp. Geometry, Pisa, Italy, pp. 107-116 (2005).

26. Sharma, V.: Complexity of real root isolation using continued fractions. Theor. Computer Science 409(2) (2008). Also: proceedings ISSAC'07

27. Snyder, J.M.: Interval analysis for computer graphics. SIGGRAPH Comput. Graph. 26(2), 121-130 (1992)

28. Stander, B.T., Hart, J.C.: Guaranteeing the topology of an implicit surface polygonalization for interactive meshing. In: Proc. 24th Computer Graphics and Interactive Techniques, pp. 279-286 (1997)

29. Yap, C.K.: Robust geometric computation. In: Goodman, J.E., O'Rourke, J. (eds.) Handbook of Discrete and Computational Geometry, Chapter 41, 2nd edn., pp. 927-952. Chapman \& Hall/CRC, Boca Raton (2004)

30. Yap, C.K.: Complete subdivision algorithms, I: Intersection of Bezier curves. In: 22nd ACM Symp. on Comp. Geometry, July 2006, pp. 217-226 (2006)

31. Yap, C.K., Yu, J.: Foundations of exact rounding. In: Das, S., Uehara, R. (eds.). Proc. WALCOM 2009. Lecture Notes in Computer Science, vol. 5431, pp. 15-31. Springer, Heidelberg (2009). Invited talk, 3rd Workshop on Algorithms and Computation, Kolkata, India 